\title{
The impacts of the ALE and hydrostatic-pressure approaches on the energy budget of unsteady free-surface flows
}

\author{
G. Lipari ${ }^{\text {a }}$, E. Napoli ${ }^{\text {b,* }}$ \\ ${ }^{a}$ School of Mechanical, Aerospace and Civil Engineering, University of Manchester, Manchester, UK \\ ${ }^{\mathrm{b}}$ Dipartimento di Ingegneria Idraulica ed Applicazioni Ambientali, Università degli Studi di Palermo, Palermo, Italy
}

Received 21 February 2006; accepted 24 October 2007

Available online 1 November 2007

\begin{abstract}
This paper focuses on the energy budget in the calculation of unsteady free-surface flows on moving grids with and without using the 'arbitrary Lagrangian-Eulerian' (ALE) formulation or hydrostatic-pressure assumption. The numerical tool is an in-house general-purpose solver for the unsteady, incompressible and homogeneous Navier-Stokes equations in a Cartesian domain. An explicit fractionalstep method and co-located finite-volume method are used for the second-order accurate integrations in time and space. The test cases are nonlinear and linear irrotational standing waves, which allow to characterise the impacts of an ALE or Eulerian formulation with moving grids by comparison with the anticipated energy conservation. The study is also extended to viscous waves for varying waveheight-to-water-depth and basin aspect ratios. The Eulerian viewpoint produces marked overdamping as early as in the first wave period for the range of relative wave heights $\eta_{0} / h>0.01$, where $\eta_{0}$ is the wave semi-amplitude and $h$ is the undisturbed water depth. The hydrostatic calculations misrepresent the evolution of the potential and kinetic energies for $h / L>0.1$, where $L$ is the basin length, with spurious modes arising from different initial conditions.
\end{abstract}

(c) 2007 Elsevier Ltd. All rights reserved.

\section{Introduction}

The moving interface between water and atmosphere is a key feature in environmental hydraulics. The numerical techniques representing the free-surface motion are influenced by the way the domain discretisation is performed, and a wealth of contributions has been dealing with this topic in the past decades.

Based on the broad classification given by [1], three major groups can be identified. The first one (so-called fixed-grid Eulerian techniques) draws a computational domain that includes both interfacing fluids, cover them with a single mesh and then evaluate the emptiness or fullness of the cells with mass-less tracers [2] or an appropriate scalar [3]; such approach is able to handle highly-distorted shapes, but the information on the interface position is not immediately available from grid points stored as geometric

\footnotetext{
${ }^{*}$ Corresponding author.

E-mail address: napoli@idra.unipa.it (E. Napoli).
}

variables. The second one (so-called front-fixing techniques) solves the equations on a fixed domain for the aqueous domain by employing a time-dependent mapping into body-fitted curvilinear co-ordinates. The third one (so-called front-tracking techniques) creates a mesh for the aqueous domain only, let the boundary be displaced in compliance with the governing equations, and periodically regenerates the whole grid so that the interface is always a gridline (references are listed later on); the interface position is thus directly computed within the time-integration cycle but, on the downside, an excessive grid distortion can corrupt the computational accuracy in the interior domain. (So far, this approach has been tested with single-valued surfaces, although its amplification to overturning waves has been envisioned and is an area of current research [4].) The formulation used in this paper belongs to the latter group. Numerical techniques blending the features of different approaches have also been devised - for example in [5], where a split-merge mechanism is applied to the near-surface computational nodes. 
To take into account the computational effects of translating grids which adjust to the free-surface motion, the governing equations in the continuum can be written according to the so-called Arbitrary Lagrangian-Eulerian (ALE) approach. Instances of ALE handling found in the literature review can be grouped into three main categories. The first tack proceeds from the straightforward application of the relative-motion kinematics to the evaluation of time derivatives, as done for instance in [6]. The second one more extensively proceeds from the co-ordinate transformations theory, allowing the transformed co-ordinate system to depend on time and yielding the relevant differential equations, as found in $[7,8]$. The third one recalls the integral formulation of the governing equations, as done by $[9,10]$. In particular, combinations between the ALE approach and finite-volume techniques are reported in $[6,8,9,11-15]$.

Three-dimensional models have been used for predicting free-surface flows for a long time now. Based on scaling arguments, the earlier ones invoked the hydrostatic-pressure assumption (hydrostatic 3D models) in order to simplify the vertical Navier-Stokes equation and avoid the computational burden caused by the pressure-velocity decoupling in incompressible fluids (among others [1618]). Whereas this is quite a sensible approximation in shallow and nearly regular domains, in several environmental applications the scaling assumptions may locally fail, for example, on account of the domain shape where significant vertical accelerations can easily be induced by closing boundaries. The importance of these limitations is witnessed by a number of later models, developed until recently, considering the full vertical momentum balance at an increased computational cost (fully-3D models), some of which extended previous hydrostatic 3D models; see [6,19-23].

Based on this research, it has been acknowledged that, when dealing with moving-boundary problems, the ALE formulation is an improvement over the purely Eulerian one, and that (irrespective of the interface handling) using the hydrostatic-pressure assumption beyond 'certain limits' produces inaccurate results. Still, an ad hoc numerical study may be purposeful firstly to study the trends with which simplified approaches cause increasingly significant inaccuracies, and secondly to identify the conditions in which numerical simulations should strictly include an ALE approach and complete pressure handling. Further, whereas previous work has widely recognised that the Eulerian formulation with moving grids generates artificial mass sources and fictitious velocities, to our knowledge its impact on energy conservation in prolonged unsteady calculations has not been given quantitative evidence and characterised.

To address these points, we performed the analysis of the energy budget in periodical, linear as well as nonlinear, irrotational standing waves, which is particularly appropriate since energy dissipations and differences in the oscillation period may be arguably caused by the inappropriate use of the standard Eulerian formulation and/or hydrostatic-pressure assumption. Moreover, we extended the numerical experiments to viscous standing waves to locate a point of compromise between model complexity and accuracy of results. The waves considered here are linear as well as nonlinear. While the benchmark set regards only one restricted class of waves, the results may have a bearing on other time-dependent free-surface flows.

Here, the tool is an in-house general-purpose numerical model based on the finite-volume and fractional-step methods, to solve the complete unsteady, incompressible, freesurface and homogeneous Navier-Stokes equations on moving grids in the Cartesian space.

This article is structured as follows: in Section 2, we review the analytical governing equations in integral forms, paying special heed to the free-surface handling, ALE approach and hydrostatic 3D simplification; in Section 3, we introduce the numerical schemes and algorithms to integrate in time and space; in Section 4, we benchmark and comment the results obtained for the selected standing waves. In Section 5, we draw the conclusions.

\section{Physical and analytical model}

This section deals with the unsteady incompressible 3D Navier-Stokes equations relative to a homogeneous fluid in a free-surface domain. Cartesian axes are indicated as $x_{i}$ with the $x_{3}$-axis orientated vertically upwards. The summation convention on repeated indexes is used throughout the paper.

\subsection{Free-surface modelling}

The fluid domain is thought of as a field of water columns in the horizontal plane $\left(x_{1}, x_{2}\right)$ with a single particle in contact with the atmosphere (single-valued free surfaces). The locus of fluid elements laying on the free surface at a moment $t, F\left(x_{1}, x_{2}, x_{3}, t\right)=0$, thus takes the form

$F\left(x_{1}, x_{2}, x_{3}, t\right)=\eta\left(x_{1}, x_{2}, t\right)-x_{3}$,

where the height function $\eta$ defines the instantaneous surface level.

The condition $\mathrm{D} F / \mathrm{D} t=0$ gives the evolutionary equation to the interface (the free-surface kinematic boundary condition) which can be written as

$\frac{\partial \eta}{\partial t}+u_{j} \frac{\partial \eta}{\partial x_{j}}=u_{3}, \quad j=1,2$

and, alternatively, as

$\frac{\partial \eta}{\partial t}-\frac{u_{j} n_{j}}{n_{3}}=0, \quad j=1 \ldots 3$,

where $u_{j}$ and $n_{j}$ are the $j$-th components of the velocity vector and of the outward-pointing unit vector normal to the free-surface element, respectively. Eqs. (2) and (3) are related by the description of the normal unit vector in terms of minus the normalised gradient of $F$ : 
$n_{i}=-\frac{\partial F}{\partial x_{i}}\left(\sqrt{\frac{\partial F}{\partial x_{j}} \frac{\partial F}{\partial x_{j}}}\right)^{-1}, \quad i, j=1 \ldots 3$.

Eq. (3) shows the straightforward relation between the local rate of change of the free-surface position and the local normal velocity and is used here as a governing equation. Among the reviewed literature, this expression has only been preferred by $[5,13]$.

In addition to the kinematic boundary condition, the free-surface dynamic boundary condition prescribes that forces are in equilibrium across the air-water interface $[25,26]$. Surface tension and external shearing actions are not considered in the following applications. Therefore, in the assumption that the atmospheric pressure is uniformly zero over the interface for all $t$ 's, the component of the stress vector normal to the interface yields the pressure boundary condition:

$p=2 v \frac{\partial u_{n}}{\partial n}$,

where the pressure symbol implies division by the fluid density $\rho ; u_{n}=u_{j} n_{j}$ is the fluid velocity normal to the interface; and $v$ is the kinematic viscosity. For the time being, the normal velocities are assumed to be known: see Section 3.2.3 for further details.

In addition, when $v \neq 0$, the stress-vector components tangential to the free surface yield the Neumann-type conditions for the tangential velocities $u_{s}$ and $u_{t}$ :

$\frac{\partial u_{s}}{\partial n}=-\frac{\partial u_{n}}{\partial s} ; \quad \frac{\partial u_{t}}{\partial n}=-\frac{\partial u_{n}}{\partial t}$,

where $s_{i}$ and $t_{i}$ are two chosen orthogonal unit vectors on the osculating plane normal to $n_{i}$, and $u_{s}=u_{j} s_{j}, u_{t}=u_{j} t_{j}$. (The subscript to $t$ avoids confusion with the symbol for time.) When $v=0$, null normal derivatives are imposed.

Hereinafter, the $F$ subscript indicates on-surface variables.

\subsection{Pressure decomposition}

Leaning on Expression (1), the pressure field $p$ is split into the sum of the modified pressure $q[25]$ and hydrostatic pressure, dependent on the surface position, as follows:

$p\left(x_{1}, x_{2}, x_{3}, t\right)=q\left(x_{1}, x_{2}, x_{3}, t\right)+\rho g\left[\eta\left(x_{1}, x_{2}\right)-x_{3}\right]$,

where $g$ is the acceleration of gravity. Expression (5) implies single-valued free surfaces and a bottom supporting the water column [27]. On the free surface, of course, $q \equiv p$.

\subsection{Integral governing equations: the Eulerian viewpoint}

The equations governing the motion of an infinitesimal fluid particle at $\left(x_{1}, x_{2}, x_{3}, t\right)$ are given by Newton's second law of motion, the incompressibility constraint and the kinematic boundary condition (3). From the Eulerian point of view and using Formula (5), the first two respectively read

$$
\begin{aligned}
& \frac{\partial u_{i}}{\partial t}+\frac{\partial}{\partial x_{j}}\left(u_{i} u_{j}-v \frac{\partial u_{i}}{\partial x_{j}}\right)+\frac{\partial q}{\partial x_{i}}+g \frac{\partial \eta}{\partial x_{i}}=0, \quad i, j=1 \ldots 3,(6) \\
& \frac{\partial u_{j}}{\partial x_{j}}=0, \quad j=1 \ldots 3 .
\end{aligned}
$$

The Eulerian integral equations can be recovered after integrating the differential counterparts on a fixed control volume $V$ with boundary $S$ and invoking Green's lemma. For later convenience, we delay the application of the lemma to the modified-pressure term until Formula (31). The governing set is thus read

$$
\begin{aligned}
& \int_{V} \frac{\partial u_{i}}{\partial t} \mathrm{~d} V+\int_{S}\left(u_{i} u_{j}-v \frac{\partial u_{i}}{\partial x_{j}}\right) n_{j} \mathrm{~d} S+\int_{V} \frac{\partial q}{\partial x_{i}} \mathrm{~d} V \\
& \quad+g \int_{S} \eta n_{i} \mathrm{~d} S=0, \quad i, j=1 \ldots 3, \\
& \int_{S} u_{j} n_{j} \mathrm{~d} S=0, \quad j=1 \ldots 3, \\
& \int_{S_{F}} \frac{\partial \eta}{\partial t} \mathrm{~d} S-\int_{S_{F}} \frac{u_{j} n_{j}}{n_{3}} \mathrm{~d} S=0, \quad j=1 \ldots 3 .
\end{aligned}
$$

The variable $\eta$ in the last term of Eqs. (8) is, of course, the quote of the interfacial point above each surface element $\mathrm{d} S$. The kinematic boundary condition (10) is integrated over that portion of domain boundary which belongs to the free surface.

\subsection{Integral governing equations: the ALE viewpoint}

The ALE formulation of the integral equations is best thought of as the enforcement of the conservation principles in a volume whose boundary is allowed to move free from a priori ties to the fluid motion, i.e. arbitrarily [28]. This handling retains the intermediate tone between an Eulerian approach, where the volume is fixed in space, and a Lagrangian approach, where the volume changes solely on account of the motion of the fluid elements contained inside.

The relevant integral equations can be either written from scratch [10,29-32], or derived from Eqs. (8)-(10) $[9,11]$ by superimposing the effects of the arbitrary motion of the domain $V=V(t)$ by the relationship [9]:

$$
\begin{gathered}
\frac{\partial}{\partial t} \int_{V} \phi \mathrm{d} V=\int_{V(t)} \frac{\partial \phi}{\partial t} \mathrm{~d} V+\int_{S(t)} \phi w_{j} n_{j} \mathrm{~d} S, \\
j=1 \ldots 3
\end{gathered}
$$

where $\phi$ is a generic variable and $w_{i}$ is the displacement velocity of a boundary element.

Therefore, applying Formula (11) to the local-inertia term in (8) results in the momentum equations

$$
\begin{aligned}
& \frac{\partial}{\partial t} \int_{V} u_{i} \mathrm{~d} V+\int_{S}\left(u_{i}\left(u_{j}-w_{j}\right)-v \frac{\partial u_{i}}{\partial x_{j}}\right) n_{j} \mathrm{~d} S \\
& \quad+\int_{V} \frac{\partial q}{\partial x_{i}} \mathrm{~d} V+g \int_{S} \eta n_{i} \mathrm{~d} S=0, \quad i, j=1 \ldots 3,
\end{aligned}
$$


which represent the dynamical equilibrium of the fluid enclosed in a volume whose boundary undergoes arbitrary displacements in time. We shall refer to the $u_{i}$-field as the absolute velocity and to the $\left(u_{i}-w_{i}\right)$-field as the relative velocity between fluid elements and moving boundaries.

The ALE viewpoint does not affect the free-surface equation (10), as the interface is assumed to move only on account of material displacements rather than arbitrary actions.

To maintain a consistent space representation, the volume- or space-conservation law links the rate of volume change and boundary velocity $w_{i}$ of each control volume together $[8,10,29-32,11,14]$ :

$\int_{S} w_{j} n_{j} \mathrm{~d} S=\frac{\mathrm{d} V}{\mathrm{~d} t} ; \quad j=1 \ldots 3$.

\subsection{Hydrostatic assumption and $3 D$ equations}

The well-known hydrostatic 3D equations stem from the Navier-Stokes equations by assuming that the effects of the remaining terms upon the modified-pressure gradient are negligible in the vertical equation. The vertical scalar equation thus reduces to

$\frac{\partial q}{\partial x_{3}}=0$

whereby the modified-pressure field takes the free-surface value $q\left(x_{1}, x_{2}, \eta, t\right)$, and the pressure distribution $p$ becomes hydrostatic according to Formula (5). Hence, the vertical momentum equation is replaced by Stevin's law, while the equations on axes $i=1,2$, devoid of the modified-pressure gradient term, allow the computation of the horizontal motion field.

\section{Numerical model}

\subsection{Space discretisation and integration}

The model can deal with a structured grid of non-overlapping hexahedral cells, non-orthogonal and clustered in special regions when necessary. They are numbered with the $p, q, r$ discrete co-ordinates, which mimic the Cartesian co-ordinates $x_{1}, x_{2}, x_{3}$ and span over the ranges $1 \ldots N_{p}$, $1 \ldots N_{q}, 1 \ldots N_{r}$, respectively.

The faces shared by the $(p, q, r)$ cell and the neighbouring cells having indices $(p+1, q, r),(p-1, q, r),(p, q+1, r)$, $(p, q-1, r),(p, q, r+1)$ and $(p, q, r-1)$ orderly take the names 'east', 'west', 'north', 'south', 'up' and 'down' and subscripts E, W, N, S, U, D.

The pressure and velocity unknowns are allocated in the cell centroids, while the surface positions are allocated in the centroids of the U-faces of the near-surface cells ( $\mathrm{co}$ located arrangement). Conveniently, in a computational domain with a single-valued interface, the vertices and centroids of the cells can be physically aligned along verticals.
Some notational manipulation is convenient to discretise the governing equations (9), (10), (12) and (13). Firstly, since control volumes are bounded by polygonal faces, surface integrals can be substituted with the summation of the integrals over the polyhedron's faces (with symbol $S_{f}$ )

$$
\int_{S} \phi \mathrm{d} S=\sum_{f} \int_{S_{f}} \phi \mathrm{d} S
$$

where $\phi$ is a generic variable and the summation index $f$ spans over the faces.

Secondly, the absolute-, displacement- and relativevelocity fluxes across a boundary element take the following symbols:

$\mathrm{d} \Phi=u_{j} n_{j} \mathrm{~d} S ; \quad \mathrm{d} X=w_{j} n_{j} \mathrm{~d} S ; \quad \mathrm{d} \Psi=\left(u_{j}-w_{j}\right) n_{j} \mathrm{~d} S$

with face-integrated fluxes accordingly indicated as $\Phi_{f}, X_{f}$, $\Psi_{f}$.

Finally, after introducing two shorthand symbols for the volume- and face-averaged values

$\bar{\phi}=\frac{1}{V} \int_{V} \phi \mathrm{d} V, \quad \hat{\phi}_{f}=\frac{1}{S_{f}} \int_{S_{f}} \phi \mathrm{d} S$,

and after noting that the convection terms can be written as

$\int_{S} u_{i}\left(u_{j}-w_{j}\right) n_{j} \mathrm{~d} S=\int_{S} u_{i} \frac{\mathrm{d} \Psi}{\mathrm{d} S} \mathrm{~d} S=\left.\sum_{f} S_{f} u_{i} \frac{\widehat{\mathrm{d} \Psi}}{\mathrm{d} S}\right|_{f}$,

the governing equations (12-13) and (9-10) are orderly written with renewed symbols as

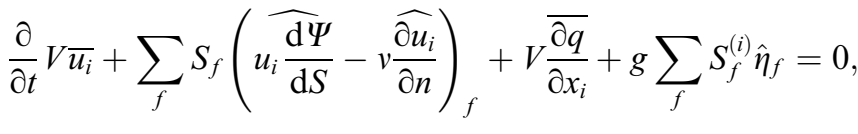

$$
i=1 \ldots 3,
$$

$\sum_{f} \mathrm{X}_{f}=\frac{\mathrm{d} V}{\mathrm{~d} t}$,

$\sum_{f} \Phi_{f}=0$

$\frac{\partial}{\partial t} S_{F} \hat{\eta}_{F}-\frac{\Phi_{F}}{n_{3}}=0$,

where $S_{f}^{(i)}=n_{i} S_{f}$ is a face projection onto the plane normal to the $x_{i}$-direction, and $\Phi_{F}$ is the absolute flux on the free surface.

By unfolding the time derivatives of Eqs. (14), Eq. (15) is inserted into them [8], thus giving the combined equations

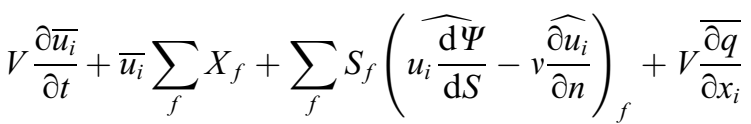

$$
\begin{aligned}
& +g \sum_{f} S_{f}^{(i)} \hat{\eta}_{f} \\
& =0 ; \quad i=1 \ldots 3
\end{aligned}
$$

with a newly-arisen second term that can be called a 'celldivergence term'. 
For computational ease, the momentum convective terms undergo the approximation [33]

$\left.\sum_{f} S_{f} u_{i} \frac{\widehat{\mathrm{d} \Psi}}{\mathrm{d} S}\right|_{f} \approx \sum_{f} S_{f} \widehat{u}_{i f} \frac{\widehat{\mathrm{d} \Psi}}{\mathrm{d} S} f=\sum_{f} \widehat{u}_{i f} \Psi_{f}$,

compatible with a second-order accurate spatial discretisation.

In the above equations, the $\widehat{\text { ace }}$-averaged values are linked to the volume-averaged unknowns by additional linear relationships expected to conform with the secondorder spatial accuracy. We used the linear formula

$\left.\hat{\phi}_{f}\right|_{p q r}=\left.\lambda_{f} \bar{\phi}\right|_{\mathrm{A}}+\left.\left(1-\lambda_{f}\right) \bar{\phi}\right|_{\mathrm{B}}$,

wherein the subscripts A, B denote the indices of the cells sharing face $f$, and the $\lambda_{f}$ coefficient is calculated on the segment joining the cell centroids and intersecting the face.

Additionally, the discrete second derivatives require formulae to handle face-averaged normal gradients. In the finite-volume spirit, the midpoint evaluation of the normal derivative on a face $f$ can be worked out on a new control volume centred on the $f$-face centroid and having volume $W_{f}$ and boundary $A_{f}$ [34]. Such a companion control volume associated to each face can be drawn by shifting the face in point normally twice, inwards and outwards, so far apart as to reach the centroids of the neighbouring cells (see Fig. 1 for a simpler two-dimensional example). Therefore, the expression of the face-averaged normal derivative is

$$
\begin{aligned}
\left.\widehat{\partial \phi}\right|_{f} & =\left.n_{f j} \frac{\widehat{\partial \phi}}{\partial x_{j}}\right|_{f}=\frac{n_{f j}}{W_{f}} \int_{W_{f}} \frac{\partial \phi}{\partial x_{j}} \mathrm{~d} W=\frac{n_{f j}}{W_{f}} \int_{A_{f}} \phi m_{j} \mathrm{~d} A \\
& =\frac{1}{W_{f}} \sum_{s} n_{f j} m_{s j} A_{f s} \hat{\phi}_{f s} .
\end{aligned}
$$

Here, the indexes $j$ and $s$ span over the Cartesian components and over the faces of the companion cell related with face $f$, respectively; $n_{f}$ is the direction of differentiation, i.e. the normal unit vector to the face $f$ of the primary cell; $m_{s}$ is the normal unit vector to each $s$-th face in the companion

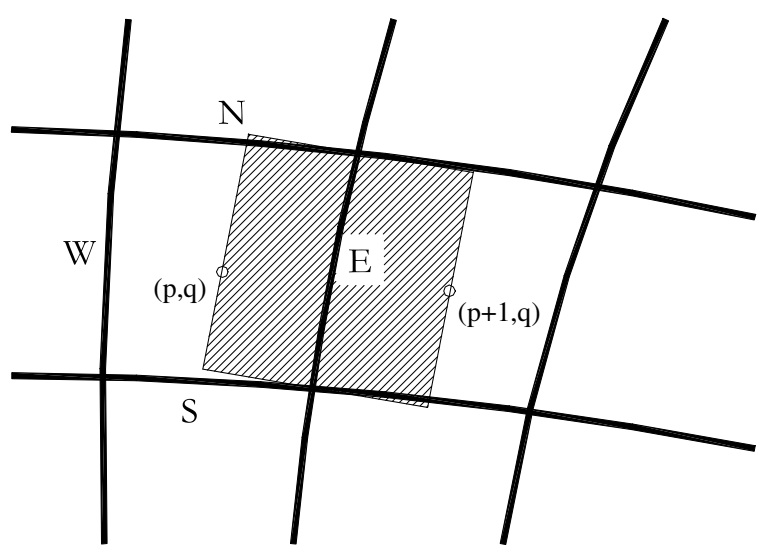

Fig. 1. A companion control volume (shaded) to compute the averaged second-derivative on the 'east' face of a primary computational cell in a finite-volume fashion (two-dimensional grid). cell; $A_{f s}$ is the $s$-th face area in the companion cell; and, finally, $\widehat{\phi}_{f s}$ is the face-averaged value of $\phi$ there. Owing to the construction of the companion cells, $\widehat{\phi}_{f s}$ does coincide with the cell-centred unknown $\bar{\phi}$ in one neighbouring cell.

On exemplifying with the 'east' face of the cell $(p, q)$ in a two-dimensional orthogonal uniformly-spaced grid, after some algebra the previous formula becomes

$$
\begin{aligned}
\left.\frac{\widehat{\partial \phi}}{\partial n}\right|_{\mathrm{E}} & =\frac{1}{W_{\mathrm{E}}} \sum_{s} n_{\mathrm{E} j} m_{s j} A_{\mathrm{Es}} \hat{\phi}_{f s}=\frac{1}{W_{\mathrm{E}}}\left(A_{\mathrm{EE}} \hat{\phi}_{\mathrm{EE}}-A_{\mathrm{EW}} \hat{\phi}_{\mathrm{EW}}\right) \\
& =\frac{A_{\mathrm{EE}}}{W_{\mathrm{E}}}\left(\hat{\phi}_{\mathrm{EE}}-\hat{\phi}_{\mathrm{EW}}\right)=\frac{A_{\mathrm{EE}}}{W_{\mathrm{E}}}\left(\bar{\phi}_{p+1, q}-\bar{\phi}_{p, q}\right),
\end{aligned}
$$

where $A_{\mathrm{EE}}, A_{\mathrm{WE}}$ and $W_{\mathrm{E}}$ are respectively the east- and westface areas and volume of the companion cell centred on the E-face of a primary computational cell. Also, $A_{\mathrm{EE}}=A_{\mathrm{Ew}}$. Moreover, $\widehat{\phi}_{\mathrm{EE}}-$ the average of $\phi$ over the east face of the cell $W_{\mathrm{E}}$ - is the volume-averaged unknown $\bar{\phi}_{p+1, q}$ (see Fig. 1); and likewise for $\widehat{\phi}_{\mathrm{EW}}$. Since the ratio $A_{\mathrm{EE}} / W_{\mathrm{E}}$ is equal to the distance between the centroids of the cells $(p+1, q)$ and $(p, q)$, one thus retrieves the standard second-order accurate finite-difference centred formula in an orthogonal uniform grid.

The overall spatial accuracy of the numerical schemes is verified in Section 4.1.

\subsection{Time discretisation and integration}

The simulated time-span is covered by steps of length $\Delta t$. Time derivatives have been discretised with a Euler scheme, giving a second-order accurate approximation at time $\Delta t\left(n+\frac{1}{2}\right)$. The terms containing spatial derivatives and the geometry are evaluated at level $n+\frac{1}{2}$, in line with [12]. The symbol $\left.\right|_{p q r} ^{n}$ is put at the right-hand side of any quantity evaluated in the cell $(p, q, r)$ and time level $n$. (No confusion with the unit vector $n$ should arise owing to the superscript position.)

The spatial-derivatives terms in the discretised momentum equations are written more compactly by posing

$\left.C_{i}\right|_{p q r} ^{n}=\left.\sum_{f} \widehat{u}_{i f} \Psi_{f}\right|_{p q r} ^{n}$

$\left.D_{i}\right|_{p q r} ^{n}=-\left.v \sum_{f} S_{f} \frac{\widehat{\partial u_{i}}}{\partial n} f\right|_{p q r} ^{n} ;$

$\left.E_{i}\right|_{p q r} ^{n}=\left.\left.\overline{u_{i}}\right|_{p q r} ^{n} \sum_{f} X_{f}\right|_{p q r} ^{n}$

$\left.G_{i}\right|_{p q r} ^{n}=\left.g \sum_{f} S_{f}^{(i)} \hat{\eta}_{f}\right|_{p q r} ^{n}$

and grouping those into

$\left.H_{i}\right|_{p q r} ^{n}=\frac{1}{\left.V\right|_{p q r} ^{n}}\left(\left.C_{i}\right|_{p q r} ^{n}+\left.D_{i}\right|_{p q r} ^{n}+\left.E_{i}\right|_{p q r} ^{n}+\left.G_{i}\right|_{p q r} ^{n}\right)$,

whence the momentum equations to be integrated in time and space are

$\left.\bar{u}_{i}\right|_{p q r} ^{n+1}=\left.\overline{u_{i}}\right|_{p q r} ^{n}-\left.\Delta t\left(H_{i}+\frac{\overline{\partial q}}{\partial x_{i}}\right)\right|_{p q r} ^{n+\frac{1}{2}}, \quad i=1 \ldots 3$. 
The incompressible pressure-velocity decoupling is overcome by a fractional-step method [35,36] that is ALEadapted as described in following subsections.

\subsubsection{Grid motion and fluxes calculation}

The volume-conservation law (15) yields the displacements fluxes $X_{f}$ as a result of the rate of change of the control volume sizes; this entirely accounts for the grid motion without computing either the vertices velocities or other displacement velocities [8,12]. Since the 'lateral' faces (E through s) are upright and the cell vertices follow the surface motion by vertical displacements, the displacements fluxes $X_{f}$ across the lateral faces are always zero, while those across the top and bottom faces are computed with the second-order accurate formula

$\left.X_{U}\right|_{p q r} ^{n}=\frac{\left.3 V\right|_{p q r} ^{n}-\left.4 V\right|_{p q r} ^{n-1}+\left.V\right|_{p q r} ^{n-2}}{2 \Delta t}-\left.X_{D}\right|_{p q r} ^{n}$.

Eq. (24) is solved columnwise (keeping the indices $p, q$ constant with $r$ stepping from 1 to $N_{r}$ ) with the conditions

$\left.X_{D}\right|_{p q r} ^{n}= \begin{cases}0, & \text { if } r=1 \text { (at the bottom); } \\ -\left.X_{U}\right|_{p q(r-1)} ^{n}, & \text { elsewhere. }\end{cases}$

One can then compute the cell-divergence terms $\left.E_{i}\right|_{p q r} ^{n}$ from (22) and, also, calculate the relative-velocity fluxes $\Psi$ in the convection terms $\left.C_{i}\right|_{p q r} ^{n}$ from their definition

$\left.\Psi_{f}\right|_{p q r} ^{n}=\left.\Phi_{f}\right|_{p q r} ^{n}-\left.X_{f}\right|_{p q r} ^{n}$.

\subsubsection{Fractional-step method: predictor step}

Eqs. (23) are first solved devoid of the modified-pressure term to obtain a fictitious vector field (pseudovelocity).

The spatial-derivative terms and the geometry information in the momentum equations are explicitly advanced in time with an Adams-Bashfort scheme. Therefore, the predictor-step equations are

$\left.\overline{u_{i}}\right|_{p q r} ^{*}=\left.\overline{u_{i}}\right|_{p q r} ^{n}-\Delta t\left(\left.\frac{3}{2} H_{i}\right|_{p q r} ^{n}-\left.\frac{1}{2} H_{i}\right|_{p q r} ^{n-1}\right), \quad i=1 \ldots 3$,

where $\left.\bar{u}_{i}\right|_{p q r} ^{*}$ is the pseudovelocity. The stability of explicit schemes with moving grids, discussed in [37], will be commented in Section 4.2.

\subsubsection{Fractional-step method: corrector step}

A velocity potential (pseudopressure) is needed to correct the pseudovelocity field into a vector field that respects both the mass conservation and dynamical equilibrium of time level $n+1$. Since here the predictor equation is explicit in time, the pseudopressure is the modified pressure times $\Delta t$, rather than a derived variable of its own. The pseudovelocity correction is easily found from the difference between Eqs. (23) and (27)

$\left.\bar{u}_{i}\right|_{p q r} ^{n+1}=\left.\bar{u}_{i}\right|_{p q r} ^{*}+\left.\Delta t \frac{\overline{\partial q}}{\partial x_{i}}\right|_{p q r} ^{n+\frac{1}{2}}, \quad i=1 \ldots 3$, while the pseudoflux correction naturally derives from the fluxes of (28):

$\left.\Phi_{f}\right|_{p q r} ^{n+1}=\left.\Phi_{f}\right|_{p q r} ^{*}+\left.\Delta t S_{f} \frac{\widehat{\partial q}}{\partial n} f\right|_{p q r} ^{n+\frac{1}{2}}$.

A Poisson-like equation for the modified pressure is then derived by working out the divergence of both sides of (28) in a finite-volume sense, i.e. summing up each Expression (29) relevant to the faces of a cell. Thence, in order to obtain a mass-conserving $\left.\bar{u}_{i}\right|_{p q r} ^{n+1}$-field, the fulfilment of the continuity Eq. (16) yields

$\left.\sum_{f} S_{f} \frac{\widehat{\partial q}}{\partial n} f\right|_{p q r} ^{n+\frac{1}{2}}=-\left.\frac{1}{\Delta t} \sum_{f} \Phi_{f}\right|_{p q r} ^{*}$,

which is to be solved in terms of cell-centred values $\bar{q}$ after having used Formula (20).

At this stage, the free-surface interface maintains the previous position rigidly. A new through-flow is allowed by specifying the Dirichlet-type boundary condition for the (face-averaged) modified pressure over the U-faces of all surface cells - see Section 2.2:

$\left.\hat{q}_{\mathrm{U}}\right|_{p, q, N_{r}} ^{n+\frac{1}{2}}=\left.2 v \frac{\widehat{\partial u_{j} n_{j}}}{\partial n} \mathrm{U}\right|_{p q N_{r}} ^{n+\frac{1}{2}}, \quad j=1 \ldots 3$.

The resulting on-surface modified-pressure gradients thus drive absolute fluxes across the free surface according to Formula (29), which are to be eventually converted into the interface's material displacement. The boundary conditions elsewhere are provided ála Neumann by Formula (29) in terms of the difference between the assigned boundary fluxes at level $n+1,\left.\Phi_{f}\right|_{p q r} ^{n+1}$ (zero on impervious, rigid walls [38]), and the computed pseudofluxes $\left.\Phi_{f}\right|_{p q r} ^{*}$.

In the present calculations, Eq. (30) has been solved for $\left.\bar{q}\right|_{p q r} ^{n+1 / 2}$ iteratively with a L-SOR algorithm [39], which is implicit along vertically-aligned cells and uses a four-colour algorithm to sweep across the computational water columns [40]. Inner iterations were stopped when the highest relative increment among the cells fell below $10^{-12}$.

On so doing, the absolute fluxes at level $n+1$ are then retrieved from Expressions (20) and (29), thus allowing one to work out the normal velocities $u_{j} n_{j}$ at the interface too. To retrieve the corrected absolute-velocity field in terms of cell-centred pressure values, Formula (28) are rather cast as though Green's lemma had been previously applied to the modified-pressure gradients of Eqs. (12). Hence

$\left.\bar{u}_{i}\right|_{p q r} ^{n+1}=\left.\bar{u}_{i}\right|_{p q r} ^{*}+\left.\frac{\Delta t}{\left.V\right|_{p q r} ^{n+\frac{1}{2}}} \sum_{f} S_{f}^{(i)} \hat{q}_{f}\right|_{p q r} ^{n+\frac{1}{2}}, \quad i=1 \ldots 3$,

which need Formula (19) to convert the computed $\bar{q}$ 's into $\hat{q}$ 's. This closes the fractional-step method procedure.

\subsubsection{Free surface step}

The free surface is now allowed to move and reach the equilibrium position field at level $n+1$. The updated positions are obtained by solving the discrete form of Eq. (17) 
$\left.\hat{\eta}\right|_{p q} ^{n+1}=\left.\hat{\eta}\right|_{p q} ^{n}+\frac{\Delta t}{2} \frac{\left.\Phi_{\mathrm{U}}\right|_{p q N_{r}} ^{n+1}+\left.\Phi_{\mathrm{U}}\right|_{p q N_{r}} ^{n}}{\left.S_{\mathrm{U}}^{(3)}\right|_{p q N_{r}}}$

where the absolute fluxes $\left.\Phi_{\mathrm{U}}\right|_{p q N_{r}} ^{n+1}$ are known from the corrector-step results (29). The projected areas $\left.S_{\mathrm{U}}^{(3)}\right|_{p q N_{r}}$ at the denominator are independent of time, since the cell vertices follow the free-surface motion by vertical displacements only. Therefore, the upper-face projections on horizontal planes, or equally the water-column bases, do not change while the free surface adjusts.

The surface quotes $\left.\hat{\eta}\right|_{p q} ^{n+1}$ are located at the centroids of the on-surface faces, whereas the cell geometry is defined through vertices: thence, the updated positions of the onsurface vertices are calculated with a bilinear interpolation. This procedure causes a minimal loss of volume whose amount is smeared back uniformly over the whole free surface, so as not to bias the surface gradients computed in the first instance.

Finally, the adjusted water columns can be re-discretised in any prescribed manner, and the geometric information at level $n+1$ can be calculated in turn.

This closes the time advancement across the $(n+1)$-th step.

\subsubsection{Segregated-integration cycle in the fractional-step method}

To summarise, the description of the ALE-adapted fractional-step algorithm is reported below:

(1) Given a solution from either the initial conditions or the previous time levels, solve the space conservation law to determine the grid-displacement fluxes and the relative fluxes - like in Section 3.2.1;

(2) Solve the predictive equation (27) for the intermediate (starred) velocity field in a domain having the interface temporarily treated as rigid - like in Section 3.2.2;

(3) Calculate the intermediate absolute fluxes across the control volumes;

(4) Solve a Poisson-like corrective equation to obtain the modified-pressure field in a domain with the rigid interface - like in Section 3.2.3;

(5) Correct the intermediate fluxes and velocities into the final divergence-free fluxes and velocities - after Eqs. (29) and (31);

(6) Update the free-surface quotes based on the throughsurface fluxes - using Eq. (32) - displace the on-surface cell vertices, and then regenerate the whole grid - like in Section 3.2.4;

(7) Re-compute all the geometric variables (volumes, areas, interpolation coefficients).

Step 1 alone embodies the essence of the ALE approach, whereby the computational overheads compared to a traditional Eulerian solution are effectively minimal (on the order of $1 \%$ CPU time). Steps $2-5$ are peculiar to the frac- tional-step segregated integration; those are replaced by the formulation of Section 3.2.6 in the case of hydrostatic assumption.

\subsubsection{Hydrostatic $3 D$ algorithm}

Eqs. (27), re-interpreted for $i=1,2$ with $u_{i}^{n+1}$ replacing $u_{i}^{*}$, yield the horizontal motion field, which allows the calculation of the absolute fluxes across the lateral (upright) faces. Such horizontal velocities and fluxes do not demand further correction.

The absolute fluxes across the top and bottom faces are then computed by solving the mass-conservation equation (16) columnwise:

$$
\begin{aligned}
\left.\Phi_{\mathrm{U}}\right|_{p q r} ^{n+1}= & -\left(\left.\Phi_{\mathrm{E}}\right|_{p q r} ^{n+1}+\left.\Phi_{\mathrm{W}}\right|_{p q r} ^{n+1}+\left.\Phi_{\mathrm{N}}\right|_{p q r} ^{n+1}+\left.\Phi_{\mathrm{S}}\right|_{p q r} ^{n+1}\right. \\
& \left.+\left.\Phi_{\mathrm{D}}\right|_{p q r} ^{n+1}\right)
\end{aligned}
$$

for $r=1 \ldots N_{r}$, with the conditions:

$\left.\Phi_{\mathrm{D}}\right|_{p q r} ^{n+1}= \begin{cases}0, & \text { if } r=1 \text { (at the bottom); } \\ -\left.\Phi_{\mathrm{U}}\right|_{p q(r-1)} ^{n+1}, & \text { elsewhere. }\end{cases}$

This terminates with a request for free-surface displacement at the top of each water column. Thus, on the domain scale, the hydrostatic 3D model appears to enforce the incompressibility constraint columnwise (or equivalently layerwise), rather than cellwise as done by the fully-3D model through the Poisson-like equation.

Further, the absolute fluxes $\Phi_{\mathrm{U}}$ and $\Phi_{\mathrm{D}}$ in each cell determine the vertical velocity components $\left.{\widehat{u_{3}}}_{f}\right|_{p q r} ^{n+1}$ at the top and bottom faces. The cell-centred values $\left.\overline{u_{3}}\right|_{p q r} ^{n+1}$ are finally retrieved by interpolation.

\subsubsection{Moving-grid special issues}

A first issue regards the interplay of the fixed-grid fractional-step method and its ALE modification (Section 3.2.3) in step 3 of the integration cycle. In fact, the pseudoflux correction, Eq. (29), contains a time-level discrepancy between either side which is solely caused by moving grids and time-changing geometry.

There, the absolute fluxes at the left-hand side belong to time level $n+1$, whereas the gradients of the pseudopressure belong to level $n+\frac{1}{2}$. Therefore, the time level of the geometry needed to derive those fluxes from the parent equation (28) can fit only either term. Consequently, Formula (29) is, to this extent, ill-posed and does not yield a 'perfectly' mass-conserving motion field for time level $n+1$. To our knowledge, this point has been envisioned only by [7].

A second issue regards the interplay of moving grids and the free-surface adjustment (Section 3.2.4) in step 6 of the integration cycle.

The grid generation redraws the division of the water column into $N_{r}$ cells, whence it does disrupt the balance of the absolute fluxes within the individual control volumes by modifying the extension of their lateral faces. Moreover, the space-conservation equation (24) cannot ever detect, nor compensate for, this modification since the displacement 
fluxes $X_{f}$ are invariably zero just across lateral faces (Section 3.2.1). This inaccuracy affects each subsequent time step through Formula (26) and might cause errors to build up over time. To our knowledge, this issue seems unreported.

Both considerations perhaps suggest that a precise fractional-step correction could be attained by solving another elliptic equation resembling (30), before delivering the absolute fluxes to the next time step. Solving this point will be addressed in subsequent work; for the time being, the impact of these issues is deemed secondary with respect to this paper's aims, provided the free surface does not undergo very rapid changes, like in the tests discussed later.

\section{Standing waves results}

Standing waves in a rectangular basin are used as benchmarks. In spite of the solution's two-dimensionality, we solved effectively three-dimensional flows that are plane in direction orthogonal to the wave, applying the free-slip condition at the boundaries parallel with that plane. Since the transversal dimension, $x_{2}$, is of no consequence in plane flows, we omit to mention it and only report the domain's horizontal and vertical sizes, along $x_{1}$ and $x_{3}$, respectively. The same kind of omission applies to the number of cells in the grid. We always solved waves having wavelength twice as large as the basin length $L$; as the latter is always taken as $10 \mathrm{~m}$, the wavenumber $k$ is 0.31416 . Unless otherwise stated, the undisturbed water depth $h$ is equal to $L$.

Examples of linear and nonlinear irrotational motion are discussed first. Then, in Section 4.4 we briefly display some calculations of the three-dimensional motion obtained from the superposition of two orthogonal waves à la Airy. In Section 4.5 , finally, we investigate viscous waves.

\subsection{A nonlinear wave}

The exact analytical solution of irrotational waves is obtainable à la Stokes as a series of increasing-order contri- butions in powers of the wave-steepness parameter $\eta_{0} k$, where $\eta_{0}$ is the wave semi-amplitude. The first-order solution is given by Airy's theory, based on a linearised form of the free-surface equation; the higher the order, the more complex is the process of deriving the solutions which eventually become unwieldy [26]. However, Agnon and Glozman found that the deep-water wave with the special steepness parameter $\eta_{0} k=0.14257$ behaves very nearly periodically for thousand of periods up to the eighth-order term of the power expansion [24].

Therefore, despite a complete analytical solution is not available, the facts that the mechanical energy is conserved and that the kinetic and potential energies convert periodically into one another enable one to monitor the behaviour of the computed energy. The bottom does affect the steepness of a wave retaining the same properties, but enough a deep basin allows exploiting the information connected to that wave at any rate [Agnon, personal communication].

To this end, we solved a wave with $\eta_{0}=0.45381 \mathrm{~m}$. The expected period having a steepness correction is $1.00253 T$, where $T$ is the first-order wave period. Hence, the deepwater period $2 \pi / \sqrt{\mathrm{kg}}$ is $3.5798 \mathrm{~s}$, while the actual one, as from Formula (36), is $3.5865 \mathrm{~s}$. A time step of $10^{-4} \mathrm{~s}$ guarantees that the results are independent of it - see also Section 4.2 for further discussion. The grid contains $32 \times 64$ cells.

The long-term behaviour of the computed energy is shown in Fig. 2 through the relative change of mechanical energy, $[E(t)-E(0)] / E(0)$. As small free-surface instabilities are known to build up and eventually generate growing oscillations after several periods, applying a smoothing function to the free-surface elevations is recommended [41]. Here a filter

$\tilde{\eta}_{i}=a \eta_{i-1}+(1-2 a) \eta_{i}+a \eta_{i+1}$

with as remarkably small a weight, $a$, as $5 \times 10^{-5}$ can prevent the onset of unbounded modes for at least 20 periods.

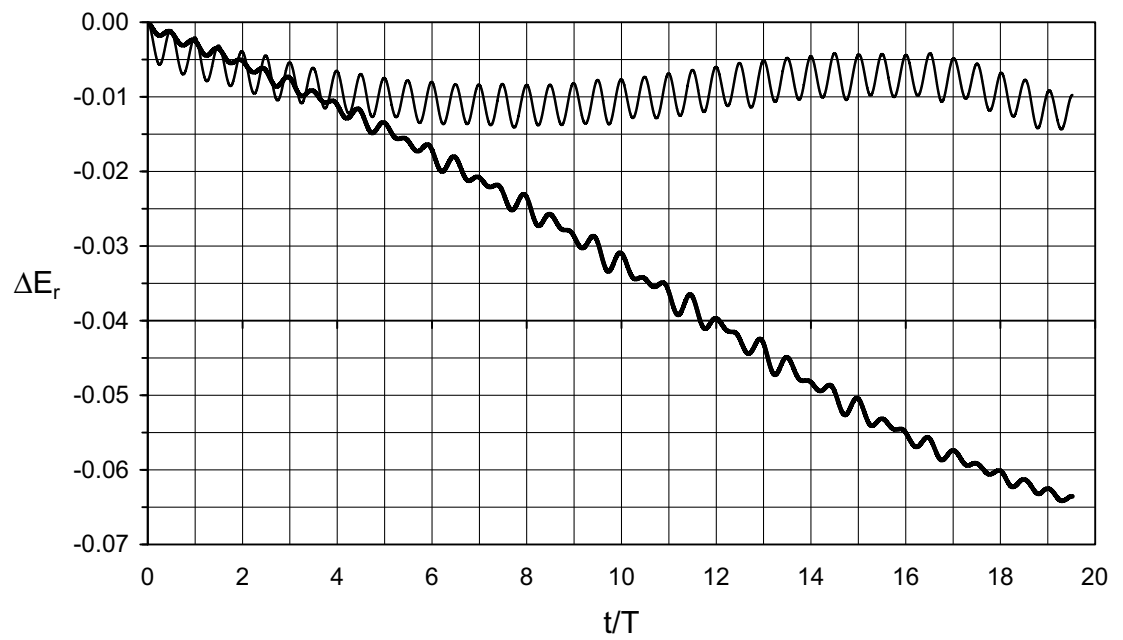

Fig. 2. Agnon-Glozman wave: energy conservation and ALE approach. History of the relative loss of the computed mechanical energy per unit mass, $\Delta E_{r}(t)=[E(t)-E(0)] / E(0) . \eta_{0}=0.45381 \mathrm{~m}, k=3.14159 \mathrm{~m}^{-1}$. Bold line: uniform grid; thin line: refined grid. Time in periods. 
The content of mechanical energy as a whole is affected by a deficit compared to the theoretically conserved value, as also observed by [13]. Noticeably, when smoothing out the free surface, the eventual outcome of the energy evolution is quite sensitive to the flow resolution achieved through the grid configuration. In particular, on the one hand, the relative energy deficit oscillates in a bounded manner within the region of $-0.9 \pm 0.5 \%$ up to nearly 20 periods when vertically-refined water columns capture the near-surface motion field more accurately. (The smallest cell is 10 times as thin as the largest one.) The results worked out on a uniform grid, also shown, on the other hand, yield a loss of comparable magnitude up to four wave periods, only to maintain a decaying trend and reach a value up to six times as large near $t=20 T$.

Fig. 3 details Fig. 2 by plotting the domain-averaged potential $(P)$ and kinetic $(K)$ energies with respect to the undisturbed water level, as they evolve during the first twelve periods starting from a quiescent state. Here, the grid is refined in the vertical direction. Both energies are always in phase opposition, the maxima of the kinetic energy and the minima of potential energy are wellbehaved. The progression to the longer term appears to consist in the slow accumulation of a residual kinetic energy and reduction of potential energy - interestingly, regardless of the interface smoother (35), that mainly extends the lapse over which well-behaved results are obtained. We leave it as a matter of future investigation to determine how far this can be determined by the issues noted in Section 3.2.7. For the time being, no additional smoothing is needed for short enough calculations, like those of the ensuing sections.

The solver's spatial order of convergence is then checked by halving the grid size using $16 \times 16$ upto $128 \times 128$ uniformly-spaced cells. The time step is $10^{-3} \mathrm{~s}$, and the free-surface smoother is turned off. Fig. 4a shows the relative deficit of the mechanical energy $[E(t)-E(0)] /$
$E(0)$ at $t=T / 8$ for each setting, which follows a secondorder convergence law. The temporal order of convergence is shown in Fig. 4b, where the same test quantity is plotted for four time steps decreasing from 0.1 to $0.01 \mathrm{~s}$ on grids having $64 \times 64,128 \times 128$ and $256 \times 256$ uniformlyspaced cells. The second-order convergence becomes fully evident over the entire range of time steps with the finest grid, where spatial/temporal error overshadowing is avoided.

Finally, in order to highlight the difference between the ALE and Eulerian approaches, the Agnon-Glozman wave is simulated with the Eulerian equations in the $32 \times 64$ grid too, while leaving the other integration specifications unchanged. The Eulerian potential and kinetic energies of Fig. 5 are clearly damped by a substantial spurious dissipation which reduces the mechanical energy by some $10 \%$ in 2 periods and $30 \%$ in just four periods. This indicates that the spurious velocities generated by disregarding the grid motion counter the achievement of the anticipated velocities and reduce the content of kinetic energy quite early. The behaviour in viscous conditions is discussed in Section 4.5.

\subsection{Linear wave in a deep basin}

The first-order wave theory has then been used to test the motion field in detail, as done by $[9,21,23,32,42]$. The field solution can be found, for example, in [21]. The mathematical prerequisites of this solution are $\eta_{0} / h \ll 1$ and $\eta_{0} k \ll 1$. The relevant dispersion relation is

$c=\sqrt{\frac{g}{k} \tanh k h}$,

which is here used as a convenient 'shallowness index' by varying the basin depth and contrasting the computed celerity with the value $\sqrt{g h}$, which well applies to 'shallow waters'.

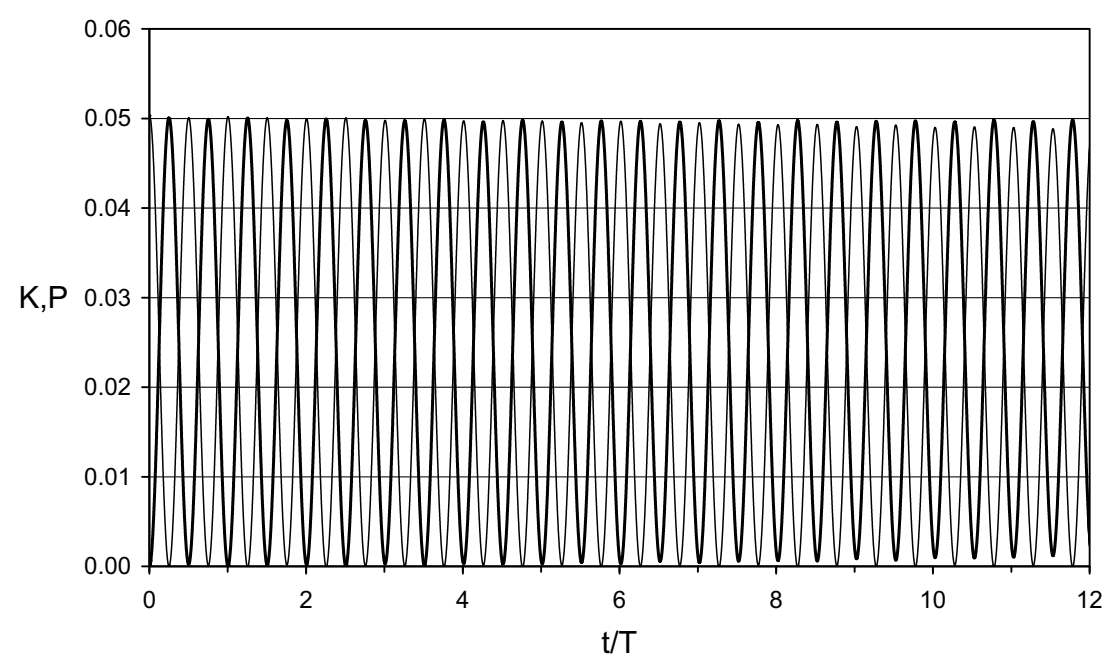

Fig. 3. Agnon-Glozman wave: energy conservation and ALE approach. Evolution of domain-averaged kinetic and potential energies per unit mass $K=K(t), P=P(t) . \eta_{0}=0.45381 \mathrm{~m}, k=3.14159 \mathrm{~m}^{-1}$. Bold line: kinetic energy; thin line: potential energy. Time in periods, energy per unit mass in meters. 

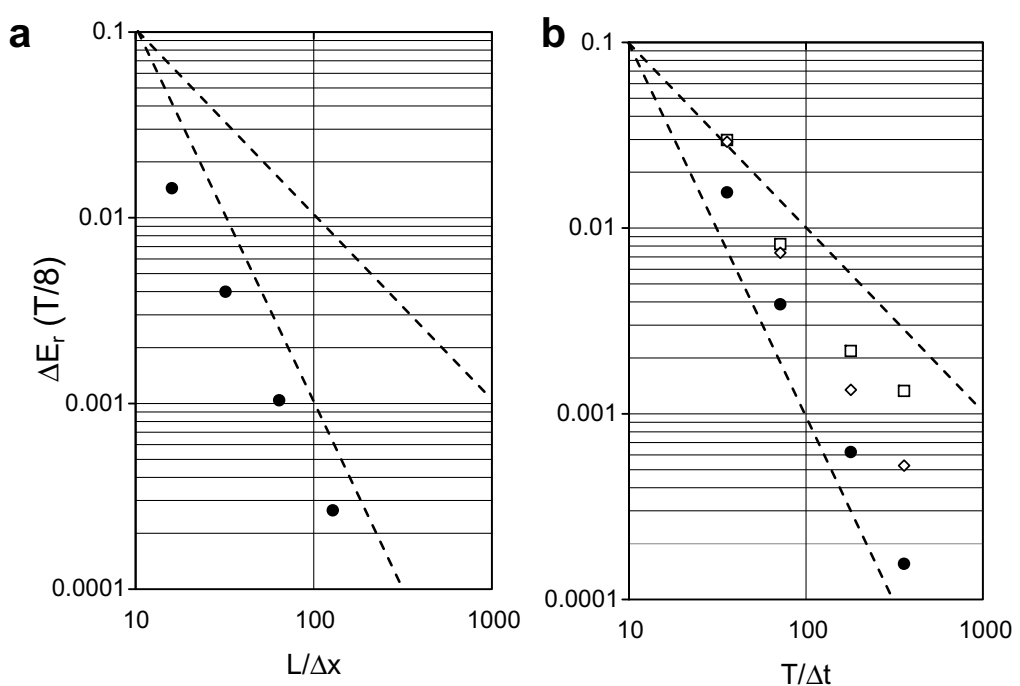

Fig. 4. Agnon-Glozman wave: spatial and temporal convergences for the mechanical-energy relative deficit $\Delta E_{r}(T / 8)$. (a) Grid-size halving from $16 \times 16$ to $128 \times 128$ cells with $\Delta t=10^{-3} \mathrm{~s}$; (b) Time-step refinement within the interval $\Delta t=0.1-0.01 \mathrm{~s}$ with $64 \times 64$ (squares), $128 \times 128$ (diamonds), $256 \times 256$ (circles) cells. First- and second-order slopes indicated.

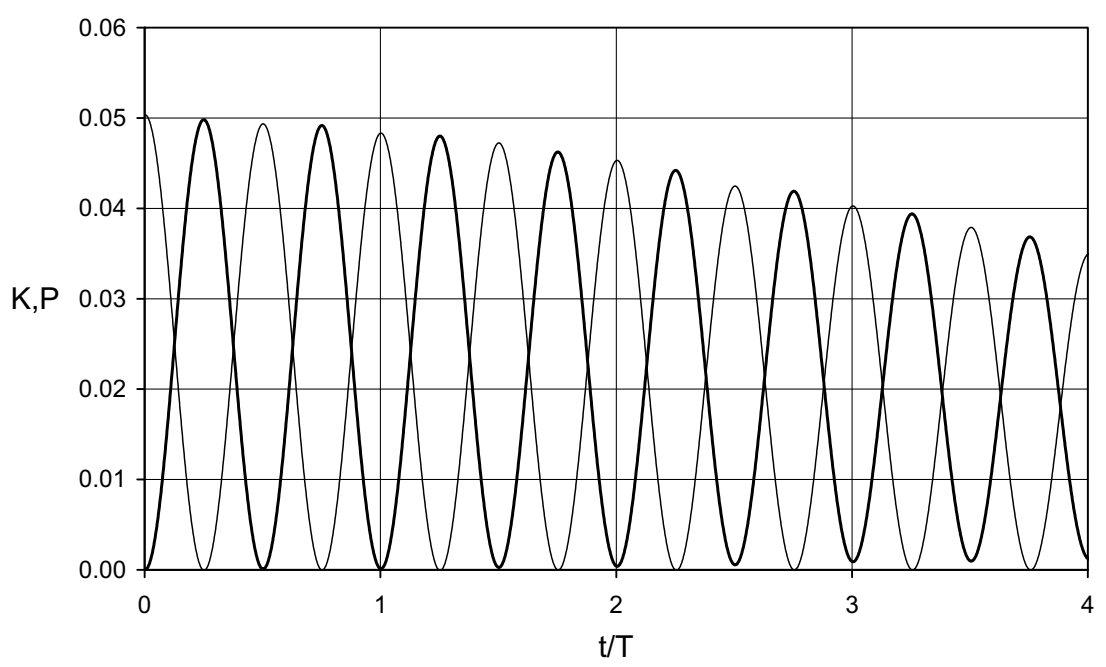

Fig. 5. Agnon-Glozman wave: energy conservation and Eulerian approach. Evolution of domain-averaged kinetic and potential energies per unit mass $K=K(t), P=P(t) . \eta_{0}=0.45381 \mathrm{~m}, k=3.14159 \mathrm{~m}^{-1}$. Bold line: kinetic energy; thin line: potential energy. Time in periods, energy per unit mass in meters.

Here, the motion is driven by a wave with $\eta_{0}=0.1 \mathrm{~m}$ $\left(\eta_{0} / h=0.01\right)$. From Formula (36) the celerity $c$ is $5.578 \mathrm{~m} / \mathrm{s}$ and the period $T$ is $3.586 \mathrm{~s}$ long. Since the shallow-water celerity is $9.905 \mathrm{~m} / \mathrm{s}$, the case proves suitable for testing the fully-3D model (F3D, hereinafter) and, then, observing the energy behaviour of the hydrostatic 3D model (H3D) being ill-posed. The domain is discretised with $20 \times 20$ uniform cells.

The time step is crucial to obtain an accurate kinetic energy history as early as a few periods. Fig. 6 shows the energy evolutions for $\Delta t=10^{-2}, 10^{-3}, 10^{-4} \mathrm{~s}$, where the largest time step brings about an appreciable reduction of the kinetic-energy maxima and a rapid loss of conservativeness (about $-4 \%$ at the second peak and $-6.4 \%$ at the fourth), which does not occur for $\Delta t=10^{-3}$ and $10^{-4} \mathrm{~s}$. Furthermore, the improvement between the two smallest time steps is so small that the results for $\Delta t=10^{-3} \mathrm{~s}$ can be considered virtually independent of it. As discussed in [37], the mesh motion entails destabilising effects upon the algorithm and requires a smaller time step than that allowed by a conventional Courant stability limit. Here, all the time steps correspond to Courant numbers below 0.1 .

The velocity's numerical and analytical vector fields at their maximum kinetic energy content are displayed in Fig. 7, showing an overall excellent agreement under the appreciation allowed by graphics. The surface positions at $t=T / 4, T / 2$ and 3T/4 are shown in Fig. 8, again with 


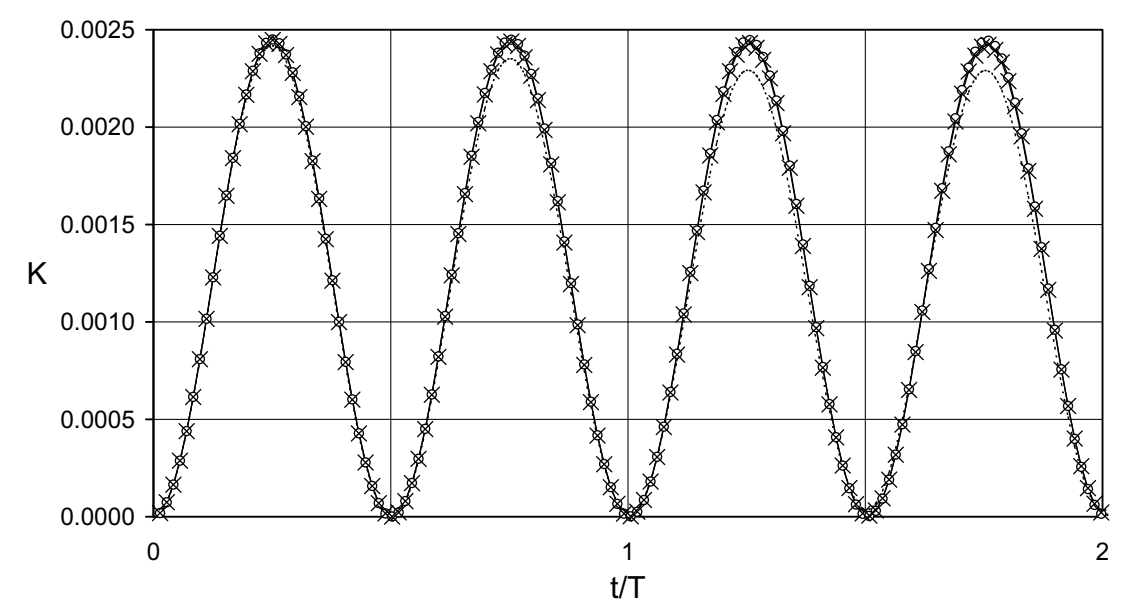

Fig. 6. Airy wave: energy conservation and integration time step. Evolution of domain-averaged kinetic energy per unit mass, $K=K(t)$. Deep basin, $\eta_{0} /$ $h=0.01$. Plain line: analytical solution; dashed line: $\Delta t=10^{-2} \mathrm{~s}$; crosses: $\Delta t=10^{-3} \mathrm{~s}$; open symbols: $\Delta t=10^{-4} \mathrm{~s}$. Time in periods, energy per unit mass in meters.
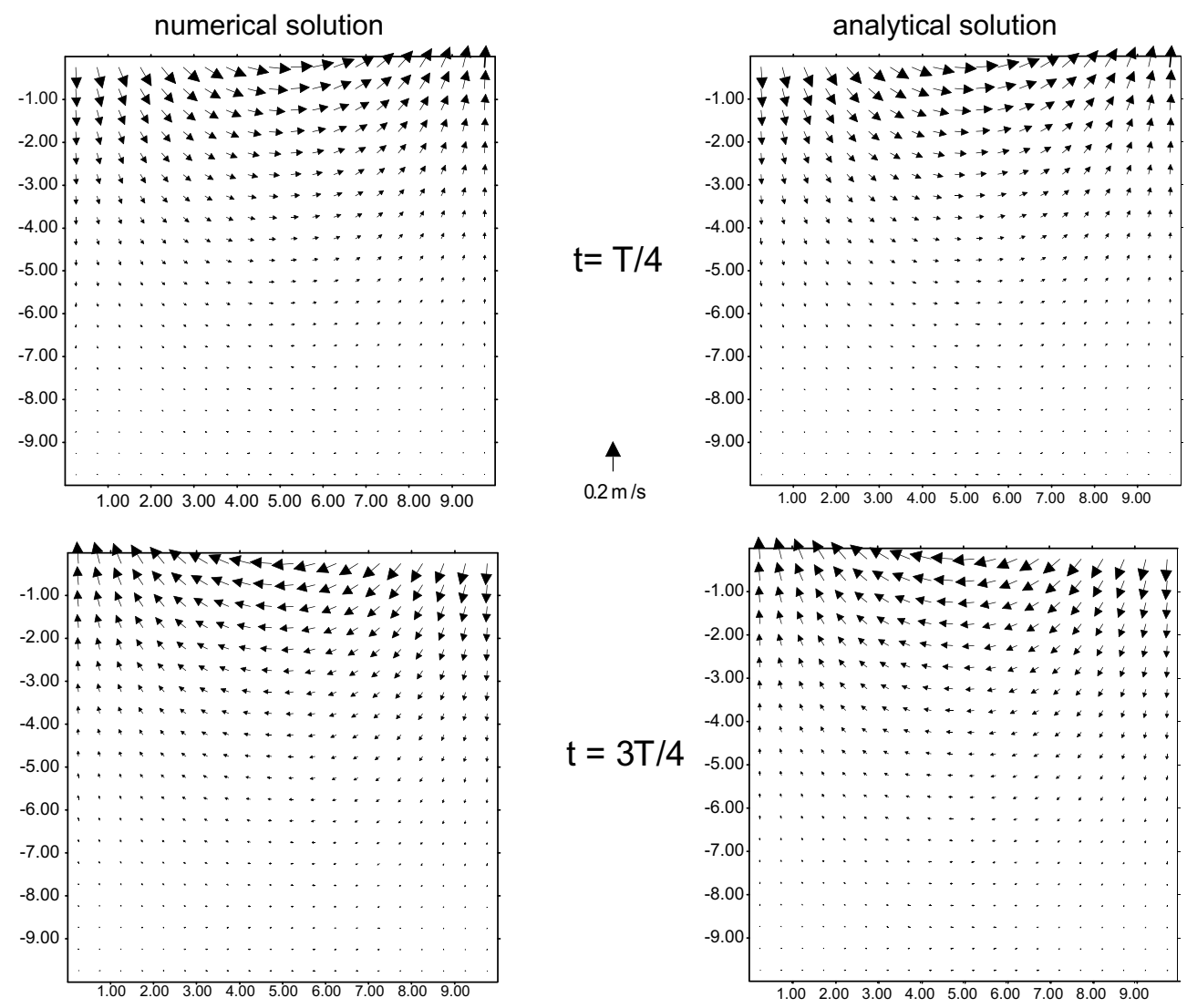

Fig. 7. Airy wave: fully-3D solver. Comparison between numerical and analytical velocity vector-fields at $t=T / 4$ and $t=3 T / 4 . \eta_{0} / h=0.01$. Lengths in meters.

excellent agreement. Finally, the time evolution of the rootmean-square errors are plotted in Fig. 9 for the $u_{1^{-}}, u_{2^{-}}, u_{3^{-}}$ velocities, which are calculated from

$\left.\mathrm{RMSE}\right|^{n}=\sqrt{\frac{\left.\sum_{p q r} V\right|_{p q r} ^{n}\left(\left.A\right|_{p q r} ^{n}-\left.N\right|_{p q r} ^{n}\right)^{2}}{\left.\sum_{p q r} V\right|_{p q r} ^{n}}}$, where $A$ and $N$ are the analytical and numerical solutions, and the sum spans all the computational cells. Also shown are the errors of the free-surface elevation $\eta$, obtained analogously from (37) after substituting the volumes $V$ with the areas of the horizontal projections of the upper faces, $S_{U}^{(3)}$, and summing over the free-surface cells only. The surface position incurs the largest loss of agreement in the first 


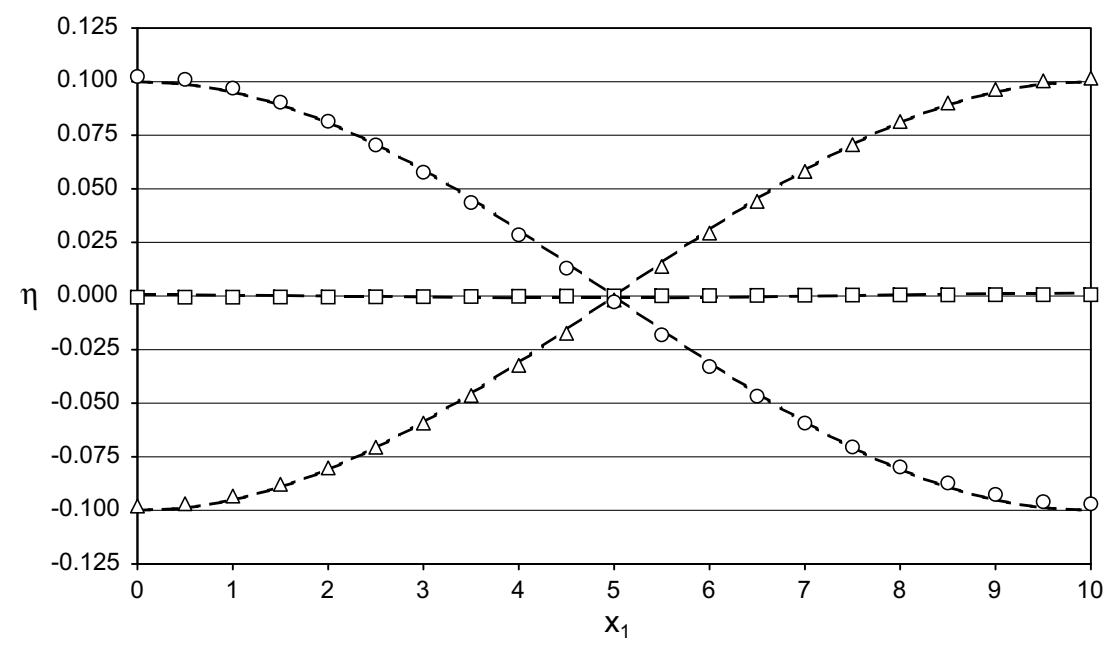

Fig. 8. Airy wave: fully-3D solver. Comparison of free-surface positions, $\eta=\eta\left(x_{1} ; t\right)$, from numerical (symbols) and analytical results (lines) at $t=T / 4$ (squares), $T / 2$ (circles), $T$ (triangles). Deep basin, $\eta_{0} / h=0.01$. Data from the first period; lengths and heights in meters.

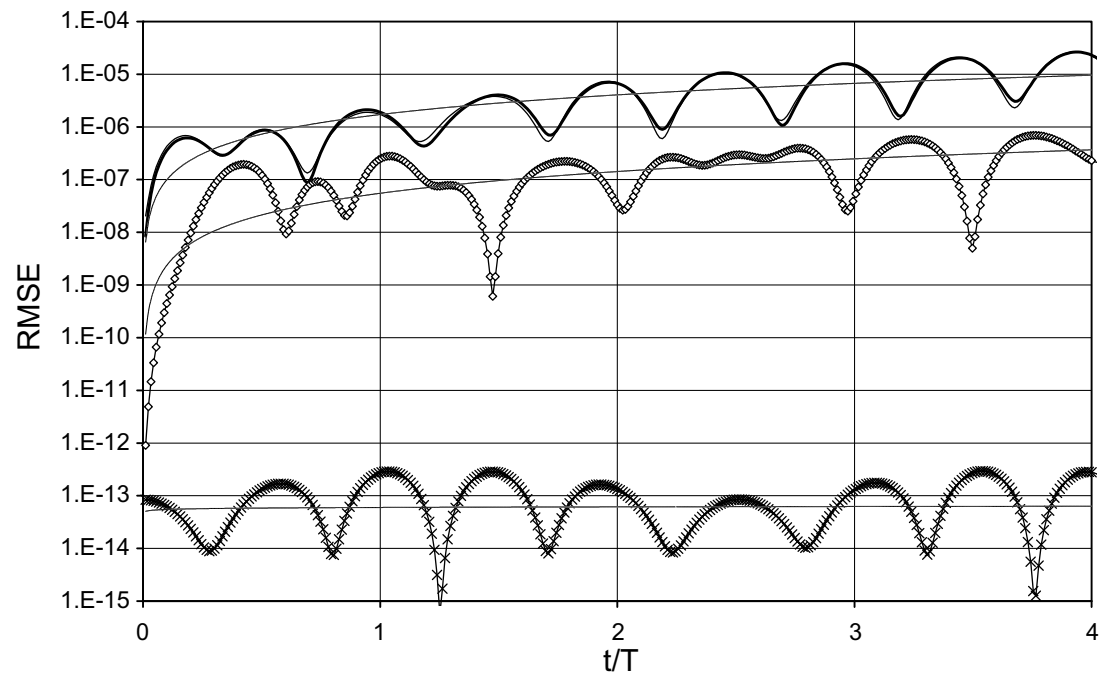

Fig. 9. Airy wave: fully-3D solver. Temporal evolution of the root-mean-square errors to velocities $u_{i}$ and $\eta$. Deep basin, $\eta_{0} / h=0.01$. Open-symbol line: surface position; plain line: $u_{1}$, wave horizontal velocity; crossed line: $u_{2}$, normal horizontal velocity; bold line: $u_{3}$, wave vertical velocity. Power-law trendlines included. Errors in $\mathrm{m} / \mathrm{s}\left(u_{i}\right)$ and $\mathrm{m}(\eta)$.

half-period, after which the index growth is far slower and stays bounded. The $u_{2}$-velocity normal to the wave plane is supposed to be null at all times, and its well-bounded RMSE gives an immediate appraisal of the numerical errors.

The same wave motion has also been solved with the illposed H3D equations (Section 3.2.6). In line with previous studies $[21,23]$, the velocity vector plots in Fig. 10 show an unphysically amplified vertical motion along the water columns. By continuity, a fairly stronger bottom stream appears in the H3D results, which suggests that an application to turbulent flows using wall functions may result in a biased calculation of the bottom friction.

Turning to the energy features, Fig. 11 shows that the expected content of the kinetic energy is severely overesti- mated (up to more than four times as high); expectedly, the wave period is underestimated down to the values prescribed by the shallow-water celerity. Perhaps surprisingly though, the performance of the H3D model is abnormally sensitive to the initial conditions as far as both amplitude and period are concerned; this is displayed in Fig. 12, where the kinetic energy evolutions are shown starting the calculations from $T / 8, T / 4$ and $T / 2$. Spurious modes appear there.

Although by deliberately focusing on the extreme of a trend, this irrotational-wave analysis characterises the consequences of using the unjustified hydrostatic assumption on the computed energy budget. The validation of the properly applied H3D solver follows in Section 4.3 . 

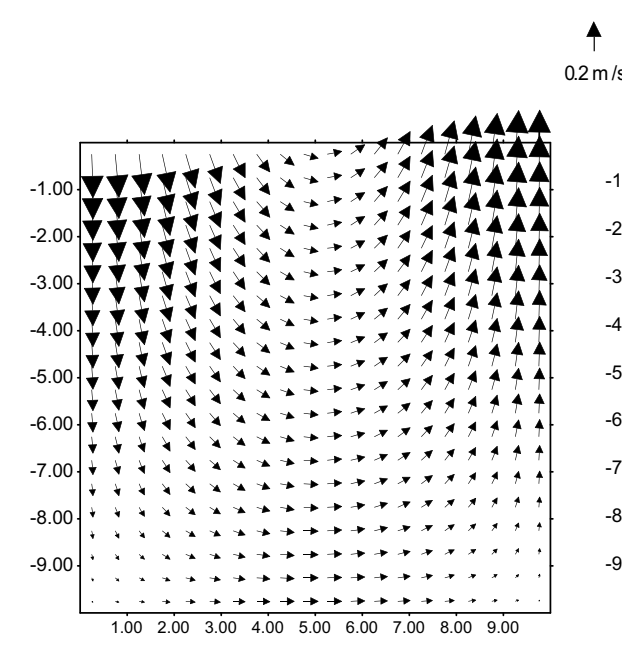

$0.2 \mathrm{~m} / \mathrm{s}$

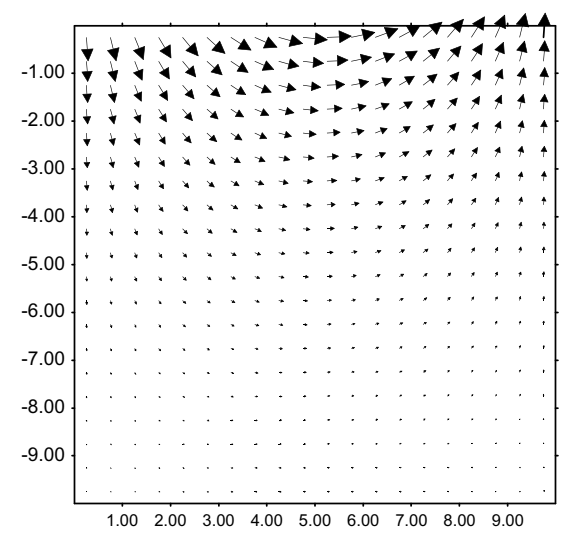

Fig. 10. Airy wave: hydrostatic approximation. Comparison between ill-posed hydrostatic 3D results (left) and fully-3D results (right) at $t=T / 4$. Velocity vector fields, $\eta_{0} / h=0.01$. Lengths in meters.

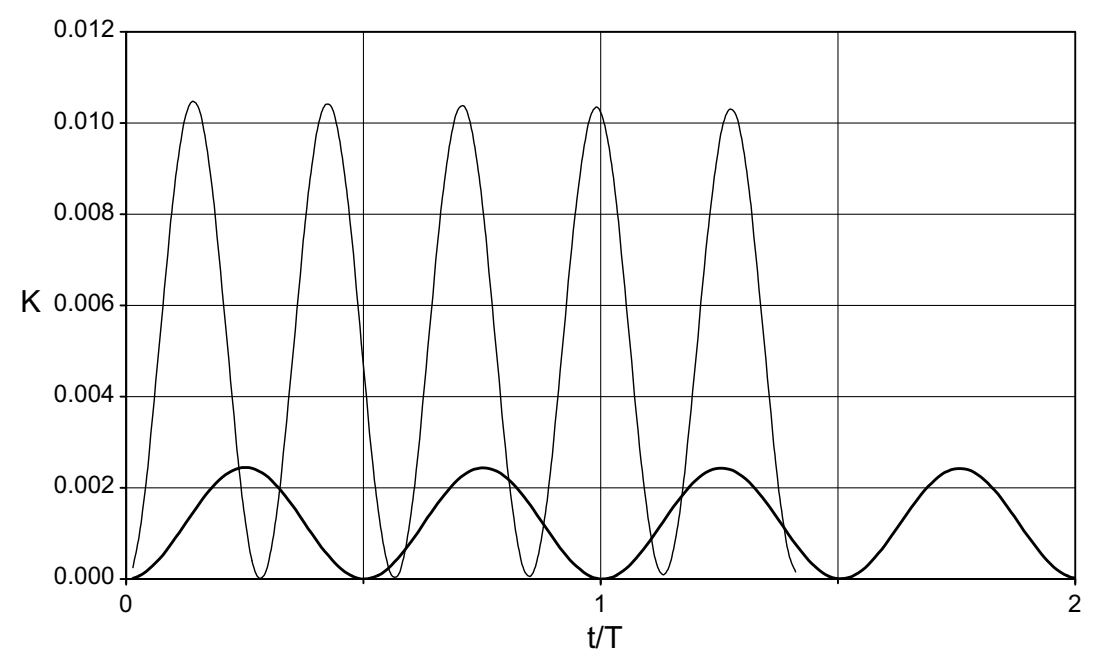

Fig. 11. Airy wave: hydrostatic approximation. Comparison between ill-posed hydrostatic 3D and fully-3D models (theoretical periods 2.019 and $3.586 \mathrm{~s}$, respectively). Kinetic energy evolution $K=K(t)$. Deep basin, $\eta_{0} / h=0.01$. Bold line: F3D; thin line: H3D. Time in periods, energy per unit mass in meters.

\subsection{Linear wave in a shallow basin}

The domain is now $1 \mathrm{~m}$ deep $(h / L=0.1)$. The wave semi-amplitude is $0.01 \mathrm{~m}\left(\eta_{0} / h=0.01\right)$. The shallow-water celerity is $3.132 \mathrm{~m} / \mathrm{s}$, whereas Formula (36) yields $3.081 \mathrm{~m} / \mathrm{s}$ ( $1.6 \%$ relative error), which correspond to wave periods of $6.386 \mathrm{~s}$ and $6.490 \mathrm{~s}$, respectively. Therefore, this case is suitable for testing the H3D equations. The discretisation parameters are the same as in the previous wave.

Indeed, unlike the deep basin of Section 4.2, the marked agreement of the two models is confirmed by the visual analysis of the vector plots at $t=T / 4$ of Fig. 13. Consistently, the kinetic-energy amplitude and wave period from the $\mathrm{H} 3 \mathrm{D}$ prediction are in much closer agreement with the F3D results than in the deep basin (Fig. 14). The aforementioned dependence on initial conditions vanishes as well (not shown). The comparison of the F3D and H3D perfor- mances with a wider range of aspect ratios $h / L$ is reported in Section 4.5 for viscous waves.

\subsection{Three-dimensional motion induced by superimposed Airy waves}

A three-dimensional irrotational motion field can be induced by the superimposition of two orthogonal smallamplitude waves in a box domain $10 \times 10 \times 10 \mathrm{~m}$ large. Here, two waves, twice as long as the sides, have semiamplitude $\eta_{0}=0.1 \mathrm{~m}$, while a uniform $20 \times 20 \times 20$ grid and $\Delta t=10^{-3} \mathrm{~s}$ have been used. The solver works in F3D-ALE mode here.

Fig. 15 shows the evolution of the domain-averaged kinetic energy per unit mass along two periods. The periodicity is again closely respected, with amplitudes slightly 


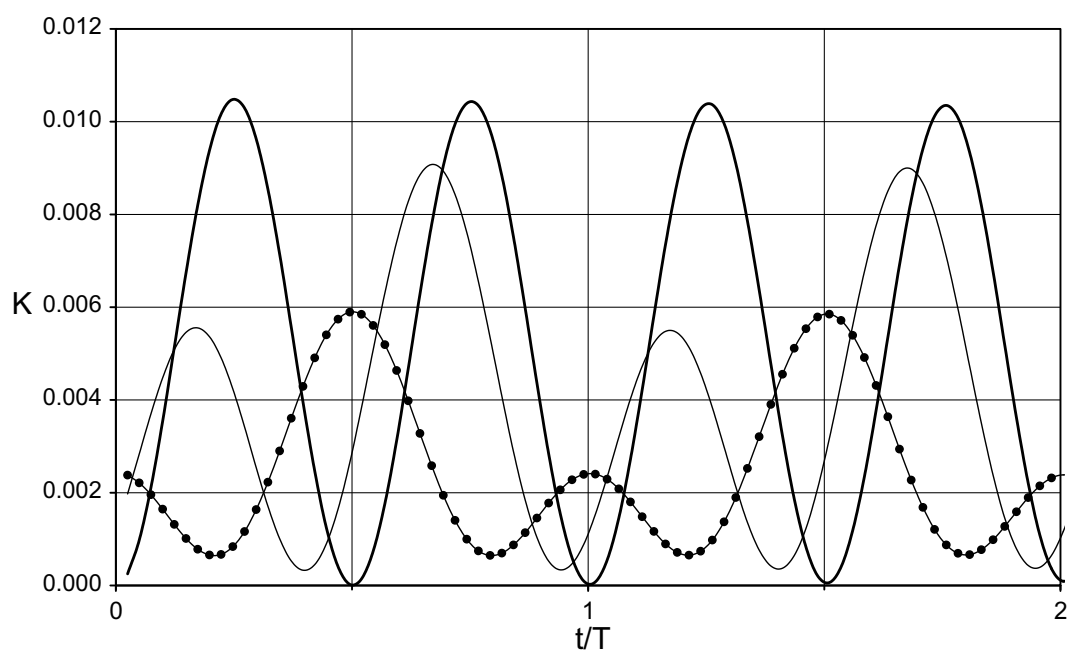

Fig. 12. Airy wave: hydrostatic approximation. Dependence of $i l l$-posed hydrostatic 3D results on initial conditions. Kinetic energy evolution, $K=K(t)$. Deep basin, $\eta_{0} / h=0.01$. Thin line: $t_{0}=T / 8$; bulleted line: $t_{0}=T / 4$; bold line: $t_{0}=T / 2$. Time in shallow-water periods, energy per unit mass in meters.

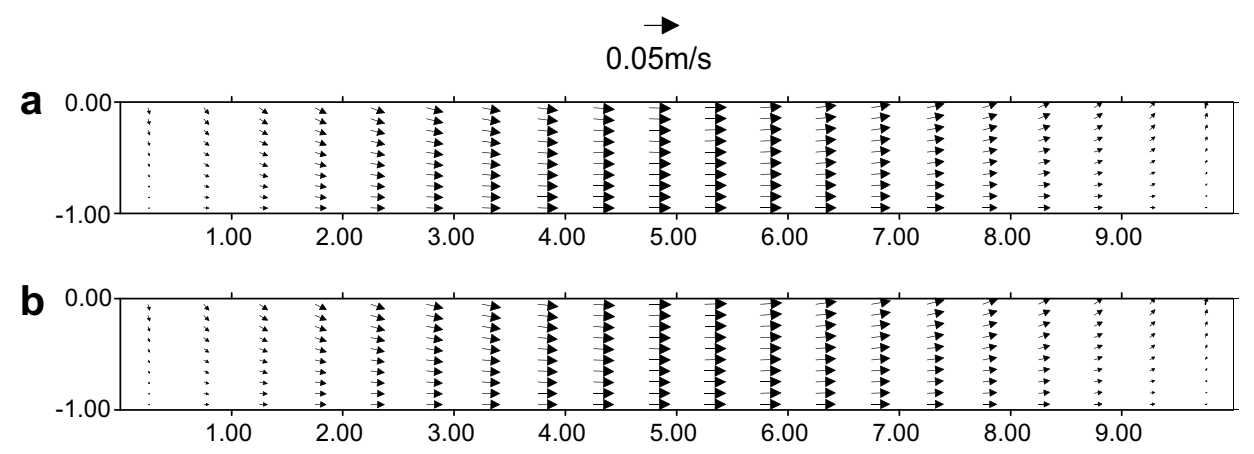

Fig. 13. Airy wave: hydrostatic approximation. Comparison between well-posed hydrostatic 3D results (a) and F3D results (b) at $t=T / 4$. Velocity vectorfields, shallow basin, $\eta_{0} / h=0.01$. Lengths in meters.

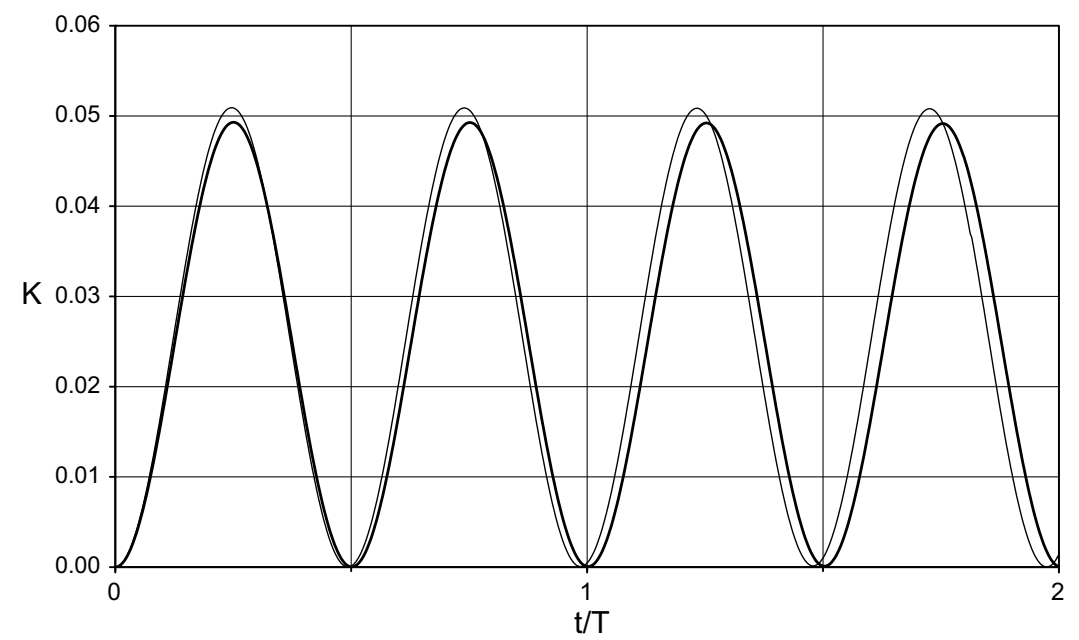

Fig. 14. Airy wave: hydrostatic approximation. Comparison between well-posed fully- and hydrostatic 3D results. Kinetic energy evolution, $K=K(t)$. Shallow basin, $\eta_{0} / h=0.01$. Bold line: F3D; thin line: H3D. Time in periods, energy per unit mass in meters.

increasing as discussed previously with respect to the solution of plane flows.
Finally, Fig. 16 displays the surface positions at quarters of period. The surface is mildly undulated at $t=T / 4,3 T / 4$ 


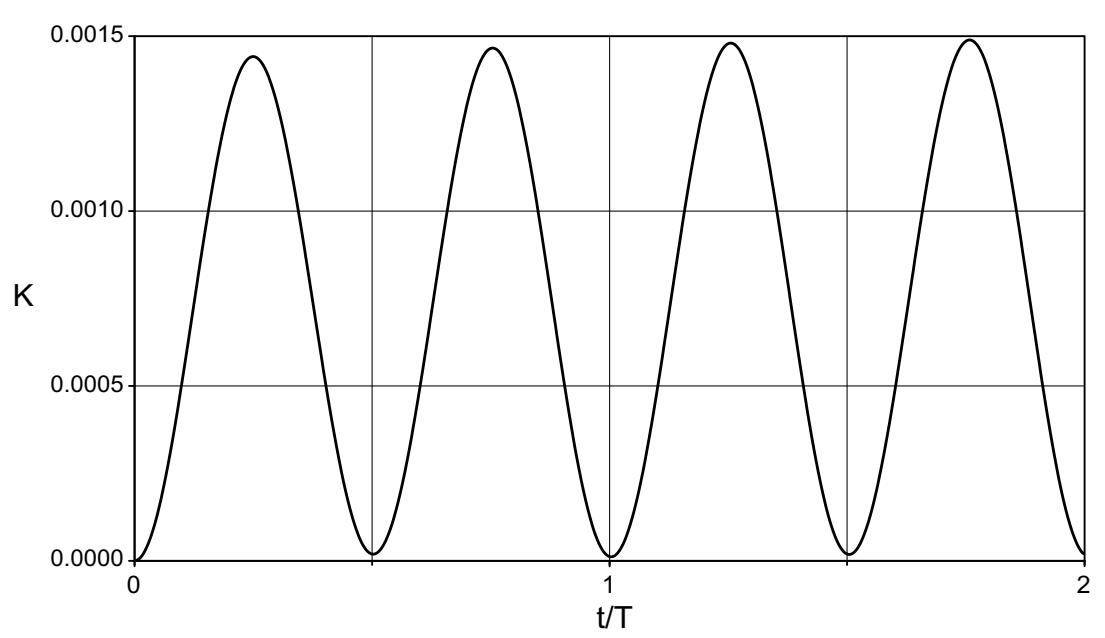

Fig. 15. Superimposition of two orthogonal Airy waves. Time evolution of the domain-averaged kinetic energy of the wave motion, $K=K(t)$. $\eta_{0} / h=0.01$. Time in periods, energy per unit mass in meters.

a
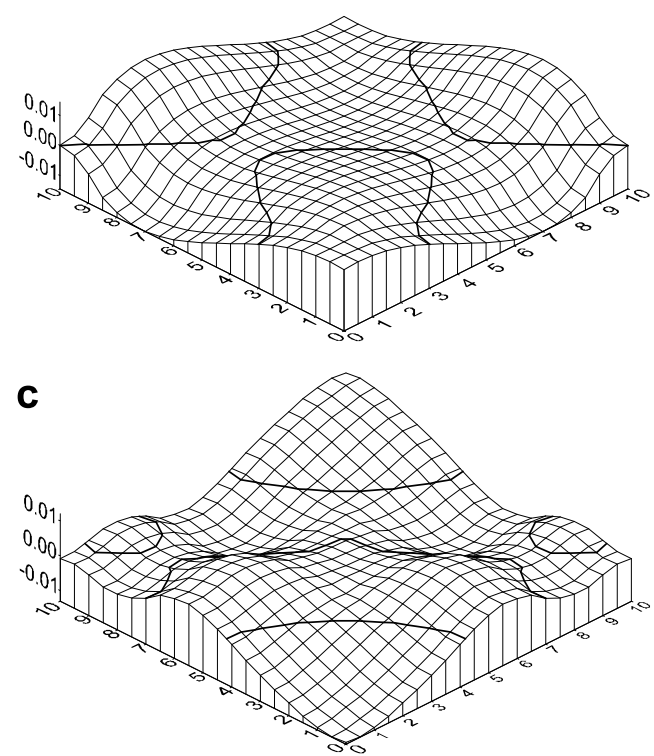

b

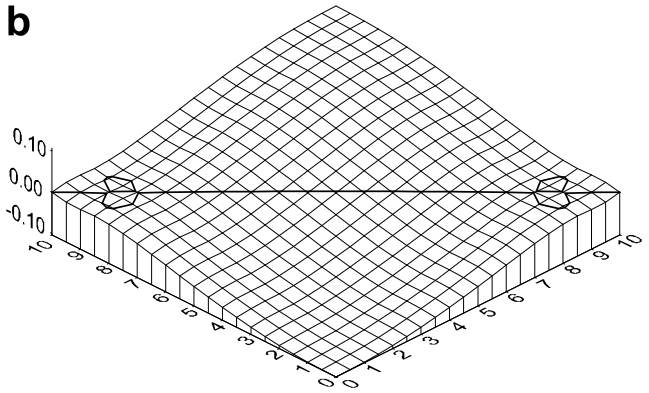

d

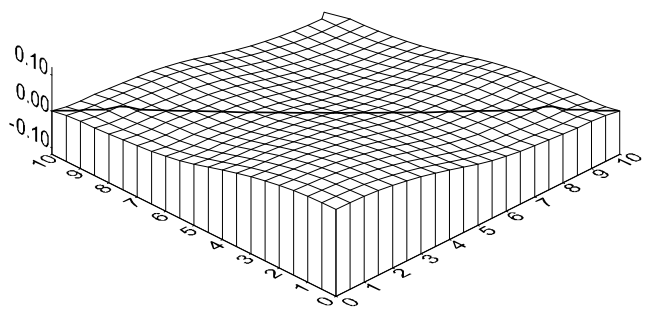

Fig. 16. Superimposition of two orthogonal Airy waves. Surface positions in the 3D irrotational motion, $\eta=\eta\left(x_{1}, x_{2}, t\right)$. (a) $t=T / 4$; (b) $t=T / 2$; (c) $t=3 T / 4$; (d) $t=T$. The vertical scales in (a) and (c) are one tenth of the others'. Isolines show the hydrostatic level $\eta=0$. Grid spacing reflects the domain discretisation. Lengths in meters.

instead of being flat as a result of the retained nonlinearity of the free-surface equation, whereas the positions at $t=T /$ $2, T$ are quite regularly antisymmetric as expected.

\subsection{Linear and nonlinear waves with viscosity}

We considered plane viscous waves having viscosities $v$ of $5 \times 10^{-3}$ and $5 \times 10^{-2} \mathrm{~m}^{2} / \mathrm{s}$ and ratios $\eta_{0} / h$ varying between 0.001 and 0.2 . The thickness of the Stokes viscous layer at the walls is of order of $(2 v / \omega)^{\frac{1}{2}}$, where $\omega$ is the wave frequency, whereby a uniform grid of $128 \times 128$ cells is adequate to solve the flow close to the wall. Also, $\Delta t=10^{-3} \mathrm{~s}$. No interface smoother is necessary. The above viscosities correspond to Reynolds parameters based on the wave celerity and basin depth of nearly $1.12 \times 10^{4}$ and $1.12 \times 10^{3}$.

Modelling the contact points between wall and free surface requires particular attention, in that they should be allowed to follow the free surface while adhering at the wall at the same and one time. Here, following [43], we applied the slip condition at the wall sides of the near-surface cells and the ordinary no-slip condition elsewhere.

The mechanical energy in a small-amplitude viscous wave is expected to decay as

$\langle E(t)\rangle=E(0) \mathrm{e}^{-2 \alpha_{T}^{t}}$,

where $\langle\cdot\rangle$ implies the average over the cycle of oscillation completed at $t ; \alpha$ is the sum of the moduli of decay account- 


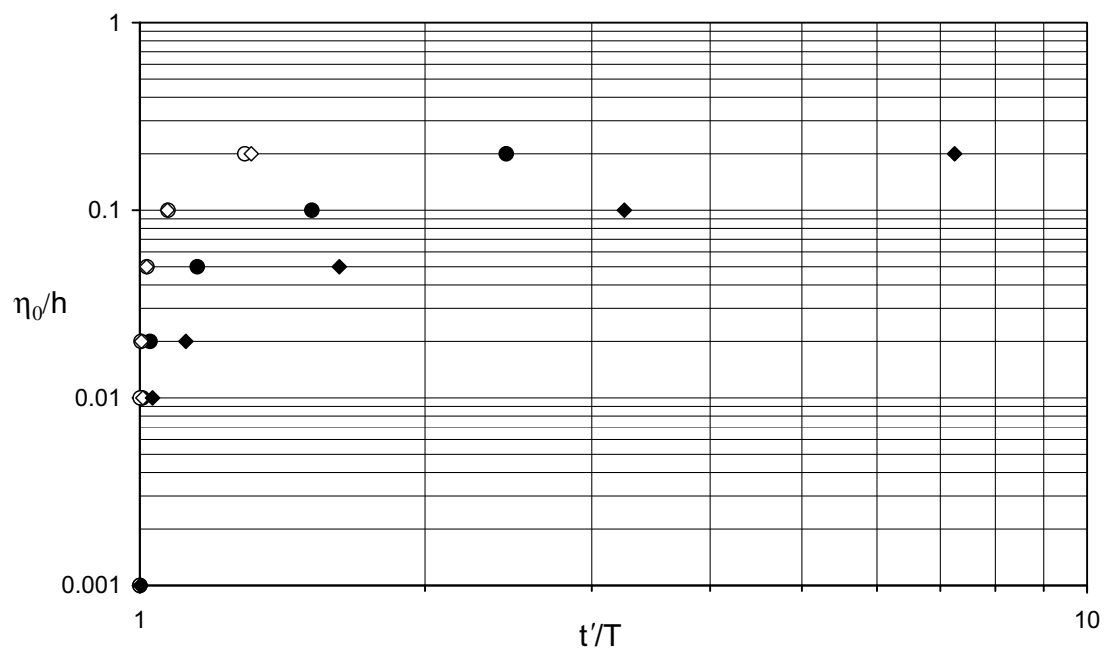

Fig. 17. Viscous standing waves: dimensionless damping time, $t^{\prime} / T$, and relative wave heights, $\eta_{0} / h$. Open symbols: ALE approach; full symbols: Eulerian. Diamonds: $v=5 \times 10^{-3} \mathrm{~m}^{2} / \mathrm{s}\left(R e=1.12 \times 10^{4}\right)$; circles: $v=5 \times 10^{-2} \mathrm{~m}^{2} / \mathrm{s}\left(R e=1.12 \times 10^{3}\right)$. Axes in log scale.

ing for frictional effects at the wall and free-surface boundary layers and in the body of the fluid. The irrotational behaviour is retrieved for $\alpha=0$.

The closed-form expression of the wall-friction modulus for standing waves in a rectangular basin is $\alpha^{\prime}=\chi(v T)^{\frac{1}{2}} B^{-1}$, where $B$ is the domain width, normal to the wave motion; $\chi$ is a function taking the specific expression $\chi=[1+$ $(\pi-k h) / \sinh (k h)] B k / 2 \pi^{\frac{1}{2}}$ when wall friction at lateral walls is neglected in the way of our computational domain [44]. The inner-fluid modulus $\alpha^{\prime \prime}$ is $2 v T k^{2}$. Both contributions outweigh that from the free surface if the latter is clean, like herein. Thereby, the chosen viscosities yield $\alpha \approx \alpha^{\prime}+\alpha^{\prime \prime}=$ $1.275 \times 10^{-2}, 4.638 \times 10^{-2}$, respectively.

Based on Formula (38), the quantity $-\ln [\langle E(T)\rangle / E(0)] /$ $2 \alpha$ should yield the dimensionless probing time $t / T=1$. Fig. 17 thus shows the values $t^{\prime} / T$ worked out with the computed residual energy ratio $\langle E(T)\rangle / E(0)$ and the theoretical modulus of decay for different $\eta_{0} / h$ 's as obtained from either the ALE or the Eulerian solver. Little algebra shows that the positive departures from the anticipated unity indicate enhanced damping. The ALE results are thus virtually insensitive to viscosity and are within phase by $2 \%$ and $7 \%$ for amplitude ratios of $\eta_{0} / h=0.05$ and 0.1 respectively; the deviation is bound to increase for larger ratios, since the wave loses the linearity for which the above estimates hold. Conversely, the Eulerian results show contained differences for ratios $\eta_{0} / h \leqslant 0.01$ only; the overdamping grows rapidly with increasing $\eta_{0} / h$ and is larger the smaller the viscosity.

Finally, Table 1 indicates the departure of the H3D results from the F3D ones when simulating a series of viscous waves with $\eta_{0} / h=0.01$ and increasing basin aspect ratio $h / L$. The losses of agreement in the wave periods and kinetic-energy maxima at $t=T / 4$ are detailed therein. The wave-period ratio decreases very nearly linearly, while that of the kinetic-energy maxima increases quadratically; both departures from unity, however, are contained within a few percent for $h / L \leqslant 0.1$. The differences obtained with a
Table 1

Viscous standing waves: ratios of hydrostatic- over fully-3D results for the wave period and kinetic energy maximum, as functions of the basin aspect ratio $h / L$

\begin{tabular}{lllllll}
\hline$h / L$ & 0.05 & 0.1 & 0.2 & 0.5 & 1 & $R^{2}$ \\
\hline$T_{\mathrm{H} 3 \mathrm{D}} / T_{\mathrm{F} 3 \mathrm{D}}$ & 0.997 & 0.984 & 0.942 & 0.764 & 0.563 & 0.994 \\
& - & 0.984 & 0.942 & 0.764 & 0.556 & - \\
$K\left(\frac{T}{4}\right)_{\mathrm{H} 3 \mathrm{D}} / K\left(\frac{T}{4}\right)_{\mathrm{F} 3 \mathrm{D}}$ & 1.007 & 1.029 & 1.124 & 1.817 & 4.312 & 1.000 \\
& - & 1.033 & 1.132 & 1.822 & 4.288 & - \\
\hline
\end{tabular}

$R^{2}$ are the coefficients of determination of a linear (periods) or quadratic (energies) trendline. $\eta_{0} / h=0.01$. Figures in Roman face: $v=5 \times 10^{-3} \mathrm{~m}^{2} /$ s; italics: $v=10^{-6} \mathrm{~m}^{2} / \mathrm{s}$.

viscosity of either $10^{-6}$ or $5 \times 10^{-3} \mathrm{~m}^{2} / \mathrm{s}$ are immaterial and are in line with the irrotational results of Section 4.2.

\section{Conclusions}

Numerical experiments were carried out to clarify the energy features of commonly-adopted modelling options, such as the Eulerian/ALE viewpoints and the fully-3D/ hydrostatic-pressure approximations, when simulating unsteady free-surface motions with regenerated (moving) grids and a $3 \mathrm{D}$ solver based on the finite-volume and time-explicit fractional-step methods. The solver is second-order accurate in time and space. We focussed on irrotational as well as viscous standing waves having relative wave heights $\eta_{0} / h$ in the range $0.001-0.2$, so as to deal with both linear and nonlinear cases. Standing waves are particularly suitable for such purposes, in that - the other discretisation parameters being kept equal - the higher the wave amplitude, the more relevant the 'cell-divergence term' peculiar to the ALE momentum equations; and, also, in that the basin aspect ratio can be changed to let the problem be described with the hydrostatic approach to a good approximation. Moreover, standing waves are a simple and fundamental flow, wherein the mechanical interplay 
of the free-surface displacement and inner motion field is immediately appreciable.

Firstly, a nonlinear irrotational periodical wave computed with the Eulerian viewpoint delivered a substantial spurious dissipation of mechanical energy. This is prevented to a large extent by solving the correct ALE equations at reduced computational overheads, which also significantly improves the expected periodical transfer between the potential and kinetic energies. The Eulerian solver produces overdamped standing waves also when the fluid viscosity is taken into account and a wider range of relative wave semi-amplitudes $\eta_{0} / h$ is investigated. The mechanical-energy contents probed at as early as the first period are consistently smaller than those expected theoretically; in particular, less viscous flows yield worse results than more viscous ones do, since in the former case the erroneous dissipation induced by the Eulerian modelling takes a larger proportion of the whole damping. The ALE solver, on the contrary, reproduces the wave damping accurately, and the departure from the theoretical anticipation for higher waves is ostensibly due to nonlinearity which, in fact, is not accounted for by the theory itself. The results obtained using either viewpoint only collapse for fairly small wave semi-amplitudes, $\eta_{0} / h \leqslant 0.01$, whereby the ALE approach appears to be more than an option even when expecting moderate grid displacements.

Secondly, we studied the consequences of neglecting the non-hydrostatic pressure by solving the fully- and hydrostatic 3D equations for irrotational waves à la Airy. Increasing-depth waves make the hydrostatic assumption deliberately ill-posed and exalt the features of how the hydrostatic 3D results depart from the fully 3D ones: in fact, the peaks of the kinetic energy are overestimated and occur at as fast a frequency as to match the "shallow water" celerity, regardless of viscosity. Further, unexpected oscillation modes arise in the kinetic-energy time evolution when the initial time is changed. The approximate H3D approach is certainly computationally less expensive than the F3D one, as it does not require solving the Poisson-like equation, elliptic in type and computationally timedemanding. (Although our estimates are not general since they depend on source-code details, we experience that the Poisson-like equation solution can take from $50 \%$ up to $80 \%$ of the runtime needed to sweep the entire time-stepping cycle, depending on whether multigrid convergence accelerators are used.) On the other hand, the F3D accuracy outperforms the H3D one in all circumstances. However, the warnings issued for ill-posed cases should not discourage the convenient application of the hydrostatic 3D model whenever its basic assumptions are reasonably well respected. The range $h / L \leqslant 0.1$ found here appears to be less strictly limiting than in the ALE/Eulerian choice.

\section{Acknowledgements}

The advice of Prof. Y. Agnon (Technion Israel Institute of Technology, Israel), Prof. A. Borthwick (University of
Oxford, UK) and Prof. P.K. Stansby (University of Manchester, UK) and the remarks from anonymous reviewers are gratefully appreciated. Both authors are indebted to Prof. G. Curto at the University of Palermo as their mentor since their early years of reading Hydraulics.

Funding from the MIUR (Italian Ministry for Education, University and Research) regarding the research on numerical simulation of free-surface flows in natural bodies of water is acknowledged. The authors' names appear alphabetically.

\section{References}

[1] Crank J. Free and moving boundary problems. Oxford, UK: Oxford University Press; 1987.

[2] Harlow FH, Welch JE. Numerical calculations of time-dependent viscous incompressible flow of fluid with free surface. Phys Fluids 1965;8:2182-9.

[3] Hirt CW, Nichols BD. Volume of fluid (VOF) methods for the dynamics of free boundaries. J Comput Phys 1981;39:201-25.

[4] Hodges BR, Street RL. On simulation of turbulent nonlinear freesurface flows. J Comput Phys 1999;151(2):425-57.

[5] Thomas TG, Leslie DC, Williams JJR. Free surface simulations using a conservative 3D code. J Comput Phys 1995;116:52-68.

[6] Zhou JG, Stansby PK. An arbitrary Lagrangian-Eulerian $\sigma$ (ALES) model with non-hydrostatic pressure for shallow water flows. Comput Methods Appl Mech Eng 1999;178:199-214.

[7] Vinokur M. Conservation equations of gas dynamics in curvilinear coordinate systems. J Comput Phys 1974;14:105-25.

[8] Demirdžić I, Perić M. Space conservation law in finite volume calculations. Int J Numer Methods Fluids 1988;8:1037-50.

[9] Sarraf S, Kahawita R, Camarero R. Three-dimensional surface water modelling using a mesh adaptive technique. Int $\mathbf{J}$ Numer Methods Fluids 1987;7:465-88.

[10] Demirdžić I, Perić M. Finite volume method for prediction of fluid flow in arbitrary shaped domains with moving boundaries. Int $\mathrm{J}$ Numer Methods Fluids 1990;10:771-90.

[11] Zhang H, Reggio M, Trepanier JY, Camarero R. Discrete form of the GCL for moving meshes and its implementation in CFD schemes. Comput Fluids 1993;22:9-23.

[12] Lesoinne M, Fahrat C. Geometric conservation law for flow problems with moving boundaries and deformable meshes and their impact on aeroelastic computations. Comput Methods Appl Mech Eng 1996;112:71-90.

[13] Mayer S, Garapon A, Sorensen LS. A fractional step method for unsteady free-surface flow with applications to non-linear wave dynamics. Int J Numer Methods Fluids 1998;28:293-315.

[14] Smith RW, Wright JA. An implicit edge-based ALE method for the incompressible Navier-Stokes equations. Int J Numer Methods Fluids 2003;43:253-79.

[15] Apsley DD, Hu W. CFD simulation of two- and three-dimensional free-surface. Int J Numer Methods Fluids 2003;42:465-91.

[16] Casulli V, Cheng RT. Semi-implicit finite difference methods for three-dimensional shallow water flow. Int J Numer Methods Eng 1992;15:629-48.

[17] Casulli V, Stelling GS. Numerical simulation of 3D quasi-hydrostatic free-surface flows. J Hydraul Eng 1998;12(7):678-86.

[18] Stansby PK. Semi-implicit finite volume shallow-water flow and solute transport solver with $k-\epsilon$ turbulence model. Int $\mathbf{J}$ Numer Methods Fluids 1997;25:285-313.

[19] Casulli V. A semi-implicit finite difference for non-hydrostatic, freesurface flows. Int J Numer Methods Fluids 1999;30:425-40.

[20] Stansby PK, Zhou JG. Shallow-water flow solver with non-hydrostatic pressure: 2D vertical plane problems. Int J Numer Methods Fluids 1998;28:541-63. 
[21] Jankowski JA. A non-hydrostatic model for free surface flows. Ph.D. thesis, University of Hannover, Germany, 1999. Available from: http://www.hydromech.uni-hannover.de/Mitarbeiter/JANKOWSKI/ diss.html [last accessed 22.04.07].

[22] Casulli V, Zanolli P. Semi-implicit numerical modeling of nonhydrostatic free-surface flows for environmental problems. Math Comput Modell 2002;36:1131-49.

[23] Koçyigit MB, Falconer RA, Lin B. Three dimensional numerical modelling of free surface flows with non-hydrostatic pressure. Int $\mathbf{J}$ Numer Methods Fluids 2002;40:1145-62.

[24] Agnon Y, Glozman M. Periodic solutions for a complex Hamiltonian system: new standing water waves. Wave Motion 1996;24:139-50.

[25] Batchelor GK. An introduction to fluid dynamics. Cambridge, UK: Cambridge University Press; 1967.

[26] Wehausen JV, Laitone EV. Surface waves. In: Flügge S, editor. Encyclopaedia of physics, vol. 9. Berlin, Göttingen, Heidelberg: Springer; 1960.

[27] Tritton DJ. Physical fluid dynamics. Oxford, UK: Oxford University Press; 1988.

[28] Hirt CW, Amsden AA, Cook JL. An arbitrary Lagrangian-Eulerian computing method for all flow speeds. J Comput Phys 1974;14: 227-53.

[29] Thomas PD, Lombard CK. Geometric conservation law and its application to flow computations on moving grids. AIAA J 1979;17:1030-7.

[30] Vinokur M. An analysis of finite-difference and finite-volume formulations of conservation laws. J Comput Phys 1989;81:1-52.

[31] Lai YG, Przekwas AJ. A finite-volume method for fluid flow simulations with moving boundaries. Comput Fluid Dyn 1994;2: $19-40$.

[32] Perot B, Nallapati R. A moving unstructured staggered mesh method for the simulation of incompressible free-surface flows. J Comput Phys 2003;184:192-214.
[33] Patankar SV. Numerical heat transfer and fluid flow. Hemisphere Publ.; 1980.

[34] Peyret R, Taylor TD. Computational methods for fluid flow. Berlin, Heidelberg, New York: Springer; 1983.

[35] Chorin AJ. Numerical solution of the Navier-Stokes equations. Math Comput 1968;22:745-62.

[36] Perot B. An analysis of the fractional step method. J Comput Phys 1993;10:51-8.

[37] Masud A. Effects of mesh motion on the stability and convergence of ALE based formulations for moving boundary flows. Comput Mech 2006;38:430-9.

[38] Veldman AEP. 'Missing' boundary conditions? Discretize first, substitute next, and combine later. SIAM J Sci Comput 1990;11:82-91.

[39] Ferziger JH, Perić M. Computational methods for fluid dynamics. Berlin, Heidelberg, New York: Springer; 2001.

[40] Rosenfeld M, Kwak D, Vinokur M. A fractional step solution method for the unsteady incompressible Navier-Stokes equations in generalized coordinate systems. J Comput Phys 1991;94:102-37.

[41] Longuet-Higgins MS, Cokelet ED. The deformation of steep surface waves on water. I. Mass transport in water waves: a numerical method of computation. Proc Roy Soc London, Ser A 1976;350:1-26.

[42] Ramaswamy B. Numerical simulation of unsteady viscous free surface flow. J Comput Phys 1990;90:396-430.

[43] Robertson I, Sherwin SJ, Graham JMR. Comparison of wall boundary conditions for numerical viscous free surface flow simulation. J Fluids Struct 2004;19:525-42.

[44] Keulegan GH. Energy dissipation in standing waves in rectangular basins. J Fluid Mech 1959;6:33-50. 